\title{
Theory for wakefields in a multizone dielectric lined waveguide
}

\author{
Changbiao Wang ${ }^{1}$ and J. L. Hirshfield ${ }^{1,2, *}$ \\ ${ }^{1}$ Omega-P, Inc., 199 Whitney Avenue, New Haven Connecticut 06511, USA \\ ${ }^{2}$ Department of Physics, Yale University, New Haven, Connecticut 06520, USA
}

(Received 21 December 2005; published 10 March 2006)

\begin{abstract}
A formal eigenmode method to solve for electromagnetic fields in a longitudinally translationally invariant multizone dielectric-lined waveguide is presented. The method is specialized to the development of wakefield theory for rectangular dielectric-lined structures which have an arbitrary number of dielectric layers. It is shown that the fields excited by a drive particle moving through the vacuum beam channel in such a structure can simultaneously include both propagating and decaying modes. The decaying modes characterize the short-range self-fields that move together with the particle, while the propagating modes characterize the long-range radiation fields or wakefields that carry energy away from the particle. It is also shown that the formal solution obtained in rectangular structures is applicable to all translationally invariant dielectric-lined waveguides, for example, cylindrical structures. Two important identities which the method relies upon are computationally confirmed for rectangular two-zone dielectric-lined structures. This theory may be employed for calculations of space charge effects, particularly for low or moderateenergy beams where self-field effects cannot be neglected.
\end{abstract}

DOI: 10.1103/PhysRevSTAB.9.031301

PACS numbers: 41.75.Jv, 41.75.Lx, 41.75.Ht, 96.50.Pw

\section{INTRODUCTION}

Recent interest has been directed towards wakefield accelerators [1-11], in which an electron (drive) bunch generates Čerenkov radiation in a dielectric-loaded waveguide (DLW)[12] and leaves the radiation behind the bunch, forming wakefields that can accelerate a closely following (test) bunch. The concept of wakefield acceleration is attractive since there is no need for $\mathrm{rf}$ power to be fed to the accelerator structure from an external source.

There is a significant number of recent publications presenting calculations of wakefields, including studies of specific configurations such as cylindrical [1-4] or rectangular DLW structures $[5,6]$. In some publications $[3,4]$, a traditional approach [12] is used that employs an inverse Fourier transform for finding field solutions, but in which the eigenvalues emerge from a contour integration that does not allow the properties of the eigenvalues to be easily identified; in other publications $[1,2,5,6]$, only real eigenvalues are identified. Consequently, all these results overlook decaying fields, and thereby provide an incomplete theoretical description.

Rectangular symmetric DLW structures have been well studied [5-9] because of their simplicity and relative ease of fabrication. Recently, a rectangular two-beam DLW acceleration structure has been proposed [10], where the arrangement of dielectric slabs is not symmetric. This necessitates development of wakefield theory for general rectangular DLW structures which have any number of dielectric layers.

There are two techniques that are often used to solve inhomogeneous differential equations with boundary-

*Email address: jay.hirshfield@yale.edu values including those that arise in radiation problems. One technique is a superposition method, while the second is an eigenfunction method; in electromagnetic theory the latter is often called the eigenmode method. The eigenmode method has been used for solving problems typified by that of excitation of fields in a waveguide from a driven current source (antenna), where all the excited modes have the same frequency, namely, that of the stationary driving source [13]. However this method cannot be used for solving wakefield problems, because all the modes excited by a moving particle have the same phase velocity but different frequencies [11]. In recognition of this, an equiphase velocity eigenmode expansion technique for cylindrical DLW structures was introduced in Refs. [2,6], wherein all the modes have the same phase velocity, equal to the drive particle velocity.

In this paper, a formal equiphase velocity eigenmode method for general longitudinally translationally invariant DLW structures is presented and is employed to obtain a complete field solution in a rectangular DLW structure, including both Čerenkov radiation fields and decaying fields. It is shown that all the eigenmodes constitute an orthogonal set which satisfies two completeness identities. It is also shown that the field solution derived for rectangular DLW structures and expressed in terms of eigenmodes is also applicable to any translationally invariant DLW structures, including cylindrical structures.

The present work can be considered to be an extension of previous work $[2,6]$ to include decaying modes, and to generalize to a translationally invariant waveguide having an arbitrary number of dielectric-lined layers - so long as eigenmodes of the waveguide structures are known. The theory may also provide an alternative method for comput- 
ing space charge effects, particularly for low or moderateenergy beams where self-field effects are not negligible.

\section{EIGENMODES AND ORTHOGONALITY}

In the eigenmode method, the electromagnetic fields to be solved for in DLW structures are expanded in terms of $2 \mathrm{D}$ eigenfunctions which are defined on the waveguide cross section, while the axial coordinate $z$ and the time $t$ are expressed through Fourier transforms. All the mode coefficients have to be calculated by exploiting mode orthogonality. Therefore, finding the orthonormality conditions for the given problem is a key step. In this section, basic field equations and orthogonality conditions for eigenmodes in an arbitrary translationally invariant system will be set up, and it will be shown that eigenvalues can be real or imaginary. Real eigenvalues correspond to propagating radiation modes produced by a moving particle in the DLW structure, while imaginary eigenvalues correspond to decaying modes that describe self-fields carried by the particle. Two identities generalized from customary waveguide systems are confirmed by computations for the equiphase velocity eigenmodes. The two identities are indispensable in solving wave equations and verifying the solutions obtained.

Suppose that $\mathbf{E}_{m n}(\mathbf{r})$ and $\mathbf{H}_{m n}(\mathbf{r})$ denote the electric and magnetic $2 \mathrm{D}$ eigenfunctions of the $n-m$ th mode that satisfy the Helmholtz equation

$$
\nabla_{\perp}^{2}\left(\mathbf{E}_{m n}, \mathbf{H}_{m n}\right)+k_{\perp m n}^{2}\left(\mathbf{E}_{m n}, \mathbf{H}_{m n}\right)=0
$$

In the above, $n$ and $m$ enumerate the eigenfunctions with respect to the $x$ and $y$ coordinates, $\nabla_{\perp}$ is the transverse gradient operator, $k_{\perp m n}$ is the transverse wave number, defined by $k_{\perp m n}^{2}=\omega_{m n}^{2} \mu \varepsilon-k_{z m n}^{2}$ where $\omega_{m n}$ is the frequency, $k_{z m n}$ is the axial wave number, and $\varepsilon$ and $\mu$ are, respectively, the permittivity and permeability. In all of what follows, ohmic losses in the conducting walls and dielectrics are neglected.

There are two basic requirements imposed on eigenmodes. First, the 2D eigenfunctions $\mathbf{E}_{m n}(\mathbf{r})$ and $\mathbf{H}_{m n}(\mathbf{r})$ multiplied by the propagator $\exp \left[i\left(\omega_{m n} t-k_{z m n} z\right)\right]$, namely $\left\{\mathbf{E}_{m n}(\mathbf{r}), \mathbf{H}_{m n}(\mathbf{r})\right\} \exp \left[i\left(\omega_{m n} t-k_{z m n} z\right)\right]$, usually known as "normal eigenmodes," satisfy the source-free Maxwell equations; and second, $\mathbf{E}_{m n}(\mathbf{r})$ and $\mathbf{H}_{m n}(\mathbf{r})$ must satisfy all transverse electromagnetic boundary conditions.

If the axial components of $\mathbf{E}_{m n}(\mathbf{r})$ and $\mathbf{H}_{m n}(\mathbf{r})$ are taken as known or generating components, the transverse components can be defined as

$$
\mathbf{E}_{\perp m n}=\frac{1}{i k_{\perp m n}^{2}}\left(k_{z m n} \nabla_{\perp} E_{z m n}+\omega_{m n} \mu \nabla_{\perp} H_{z m n} \times \hat{\mathbf{z}}\right),
$$

$$
\mathbf{H}_{\perp m n}=\frac{1}{i k_{\perp m n}^{2}}\left(k_{z m n} \nabla_{\perp} H_{z m n}-\omega_{m n} \varepsilon \nabla_{\perp} E_{z m n} \times \hat{\mathbf{z}}\right),
$$

where $\hat{\mathbf{z}}$ is the axial unit vector and $i$ is the imaginary unit.

Equations (1)-(3) are general equations for eigenmodes, applicable to both the equiphase velocity modes and the conventional equifrequency modes. It is easy to show that the first requirement is automatically satisfied; the second requirement will be used to obtain orthogonality conditions.

From Eqs. (1)-(3), we obtain

$$
\begin{gathered}
\nabla_{\perp} \times \mathbf{E}_{\perp m n}=-i \omega_{m n} \mu \mathbf{H}_{z m n}, \\
\nabla_{\perp} \times \mathbf{H}_{\perp m n}=+i \omega_{m n} \varepsilon \mathbf{E}_{z m n}, \\
\nabla_{\perp} \cdot \mathbf{E}_{\perp m n}=i k_{z m n} E_{z m n}, \\
\nabla_{\perp} \cdot \mathbf{H}_{\perp m n}=i k_{z m n} H_{z m n} .
\end{gathered}
$$

From Eqs. (2)-(5), we can obtain two alternative expressions for the cross-product $\mathbf{E}_{\perp m n} \times \mathbf{H}_{\perp p q}^{*}$ produced by $m$ - $n$th and $p$ - $q$ th modes, given by

$$
\begin{aligned}
i k_{\perp m n}^{2}\left(\mathbf{E}_{\perp m n} \times \mathbf{H}_{\perp p q}^{*}\right)= & k_{z m n} \nabla_{\perp} \times\left(E_{z m n} \mathbf{H}_{\perp p q}^{*}\right) \\
& +\hat{\mathbf{z}} \omega_{m n} \mu \nabla_{\perp} \cdot\left(H_{z m n} \mathbf{H}_{\perp p q}^{*}\right) \\
& +i\left(\omega_{p q}^{*} k_{z m n} \varepsilon E_{z m n} \mathbf{E}_{z p q}^{*}\right. \\
& \left.+\omega_{m n} k_{z p q}^{*} \mu H_{z m n} \mathbf{H}_{z p q}^{*}\right),
\end{aligned}
$$

and

$$
\begin{aligned}
i k_{\perp p q}^{* 2}\left(\mathbf{E}_{\perp m n} \times \mathbf{H}_{\perp p q}^{*}\right)= & k_{z p q}^{*} \nabla_{\perp} \times\left(H_{z p q}^{*} \mathbf{E}_{\perp m n}\right) \\
& -\hat{\mathbf{z}} \omega_{p q}^{*} \varepsilon \nabla_{\perp} \cdot\left(E_{z p q}^{*} \mathbf{E}_{\perp m n}\right) \\
& +i\left(\omega_{p q}^{*} k_{z m n} \varepsilon E_{z m n} \mathbf{E}_{z p q}^{*}\right. \\
& \left.+\omega_{m n} k_{z p q}^{*} \mu H_{z m n} \mathbf{H}_{z p q}^{*}\right) .
\end{aligned}
$$

The electromagnetic boundary conditions require that the tangential components of $\mathbf{E}$ and $\mathbf{H}$, and the normal components of $\varepsilon \mathbf{E}$ and $\mu \mathbf{H}$ are continuous on the dielectric interfaces, while the tangential $\mathbf{E}$ and normal $\mu \mathbf{H}$ components are equal to zero on the conducting walls. Considering that the third terms on the right hand sides of Eqs. (8) and (9) are identical and using divergence and curl theorems, we have

$$
\iint_{S}\left(k_{\perp m n}^{2}-k_{\perp p q}^{* 2}\right)\left(\mathbf{E}_{\perp m n} \times \mathbf{H}_{\perp p q}^{*}\right) \cdot \hat{\mathbf{z}} d S=0
$$




$$
\begin{aligned}
\iint_{S} & k_{\perp m n}^{2}\left(\mathbf{E}_{\perp m n} \times \mathbf{H}_{\perp p q}^{*}\right) \cdot \hat{\mathbf{z}} d S \\
= & \iint_{S}\left(\omega_{p q}^{*} k_{z m n} \varepsilon E_{z m n} E_{z p q}^{*}+\omega_{m n} k_{z p q}^{*} \mu H_{z m n} H_{z p q}^{*}\right) d S,
\end{aligned}
$$

where $S$ denotes the area of the waveguide cross section. Equations (10) and (11) are the general equations for orthogonality in a longitudinally uniform DLW structure, applicable to both equiphase velocity and equifrequency eigenmodes.

For the equiphase velocity eigenmodes which have a common phase velocity $v_{\mathrm{ph}}=\omega_{m n} / k_{z m n}$, we have $k_{\perp m n}^{2}=$ $\left(v_{p h}^{2} \mu \varepsilon-1\right) k_{z m n}^{2}$ and $k_{\perp p q}^{2}=\left(v_{p h}^{2} \mu \varepsilon-1\right) k_{z p q}^{2}$. From Eqs. (10) and (11), we obtain

$$
\begin{gathered}
\left(k_{z m n}^{2}-k_{z p q}^{* 2}\right) \iint_{S}\left(v_{p h}^{2} \mu \varepsilon-1\right)\left(\mathbf{E}_{\perp m n} \times \mathbf{H}_{\perp p q}^{*}\right) \cdot \hat{\mathbf{z}} d S=0, \\
\left(k_{z m n}^{2} \text { or } k_{z p q}^{* 2}\right) \iint_{S}\left(v_{p h}^{2} \mu \varepsilon-1\right)\left(\mathbf{E}_{\perp m n} \times \mathbf{H}_{\perp p q}^{*}\right) \cdot \hat{\mathbf{z}} d S \\
=v_{\mathrm{ph}} k_{z m n} k_{z p q}^{*} \iint_{S}\left(\varepsilon E_{z m n} E_{z p q}^{*}+\mu H_{z m n} H_{z p q}^{*}\right) d S .
\end{gathered}
$$

If $k_{z m n}^{2} \neq k_{z p q}^{* 2}$, from Eq. (12) we have $\int\left(v_{\mathrm{ph}}^{2} \mu \varepsilon-1\right) \times$ $\left(\mathbf{E}_{\perp m n} \times \mathbf{H}_{\perp p q}^{*}\right) \cdot \hat{\mathbf{z}} d S=0$. From this we obtain two equivalent orthogonality conditions for $v_{\mathrm{ph}}>0$, given by

$$
\begin{gathered}
\iint_{S}\left(v_{\mathrm{ph}}^{2} \mu \varepsilon-1\right)\left(\mathbf{E}_{\perp m n} \times \mathbf{H}_{\perp p q}^{*}\right) \cdot \hat{\mathbf{z}} d S \\
=\delta_{m n}^{p q}\left(k_{z m n}^{*} / k_{z m n}\right) v_{\mathrm{ph}} \Pi_{z m n}, \\
\iint_{S}\left(\varepsilon E_{z m n} E_{z p q}^{*}+\mu H_{z m n} H_{z p q}^{*}\right) d S=\delta_{m n}^{p q} \Pi_{z m n},
\end{gathered}
$$

where $\delta_{m n}^{p q}=1$ if and only if $p=m$ and $q=n$, otherwise, $\delta_{m n}^{p q}=0$; the quantity $\Pi_{z m n}(>0)$ with dimensions of a force is given by

$$
\Pi_{z m n}=\iint_{S}\left(\varepsilon E_{z m n} E_{z m n}^{*}+\mu H_{z m n} H_{z m n}^{*}\right) d S .
$$

It is seen from Eq. (13) that if $p=m$ and $q=n$, then $k_{z m n}^{2}=k_{z m n}^{* 2}$, so $k_{z m n}^{2}$ is real, and $k_{z m n}$ can be real or pure imaginary. It will be shown later that there are three different cases for a complete mode set in DLW structures: (i) all eigenvalues are real; (ii) all eigenvalues are imaginary; and (iii) both real and imaginary eigenvalues are included. In what follows, modes with real eigenvalues are referred to as "real eigenmodes," while those with imaginary eigen- values are referred to as "imaginary eigenmodes." Note that the axial wave number $k_{z m n}$ and the frequency $\omega_{m n}$ are both real for real modes while they are both imaginary for decaying modes. In this respect, fields generated by a moving charge differ from conventional initial-value or boundary-value solutions, wherein either $k_{z m n}$ or $\omega_{m n}$, respectively, would be real. For real modes, the phase velocity is the conventional phase velocity of the radiation propagating modes excited by a moving particle in the DLW structures, while for the decaying modes, the phase velocity is the velocity at which the nonoscillatory but localized decaying-mode fields move.

It should be stated that in Ref. [2] the orthogonality relations Eqs. (14) and (15) were derived for cylindrical DLW structures, but only real eigenvalues were identified because of an implicit assumption that the transverse wave number $k_{\perp m n}$ is real. As is shown in this paper, that assumption is overly restrictive.

It is seen from Eq. (14) that if the axial wave number $k_{z m n}$ is real $\left(k_{z m n}^{*} / k_{z m n}=1\right)$, corresponding to propagating radiation modes in the DLW wakefield accelerator structures, we have a quantity $\Lambda_{z m n} \equiv \int\left(v_{\mathrm{ph}}^{2} \mu \varepsilon-1\right)\left(\mathbf{E}_{\perp m n} \times\right.$ $\left.\mathbf{H}_{\perp m n}^{*}\right) \cdot \hat{\mathbf{z}} d S=v_{\mathrm{ph}} \Pi_{z m n}>0$, with dimensions of energy per unit length carried at the phase velocity. If $k_{z m n}$ is imaginary $\left(k_{z m n}^{*} / k_{z m n}=-1\right)$, corresponding to decaying modes, we have $\Lambda_{z m n}<0$. It also can be seen from Eq. (14) that there is no mode corresponding to $\Lambda_{z m n}=$ 0 . This can be easily seen for a waveguide completely filled with dielectric, where $\left(v_{\mathrm{ph}}^{2} \mu \varepsilon-1\right) k_{z m n}^{2}=k_{\perp m n}^{2}>0$ holds, leading to $v_{\mathrm{ph}}^{2} \mu \varepsilon-1 \neq 0$ and $k_{z m n} \neq 0$. The occurrence of $\Lambda_{z m n}<0$ for decaying modes does not lead to an unphysical result, since (as will be shown below), the decaying modes describe self-fields of a moving particle in the DLW structures and do not take away energy from the particle.

Based on general boundary conditions, we have shown that the eigenvalues for equiphase velocity modes can be imaginary as well as real. Below some numerical examples from dispersion equations of specific DLW structures will be given to illustrate how the real and imaginary eigenvalues come about. It should be indicated that the mode phase velocity is a real quantity in this context, since equiphase velocity modes with $\omega^{2} / k_{z}^{2}<0$ are not physical for wakefield problems, although they do exist mathematically.

It is easy to understand that there are imaginary or decaying eigenmodes for the single-zone structure comprising a waveguide completely filled with dielectric. In such a case, the real and imaginary dispersion equations in the $|\omega / c|$ vs $\left|k_{z}\right|$ plane are, respectively, given by $|\omega / c|^{2} \varepsilon_{r} \mu_{r}-\left|k_{z}\right|^{2}=k_{\perp}^{2} \quad\left(=k_{x}^{2}+k_{y}^{2} \quad\right.$ for rectangular structures) and $-|\omega / c|^{2} \varepsilon_{r} \mu_{r}+\left|k_{z}\right|^{2}=k_{\perp}^{2}$, where $c$ is the vacuum light speed, and $\varepsilon_{r}$ and $\mu_{r}$ are the relative permittivity and permeability. The real dispersion curves with both $\omega / c$ and $k_{z}$ real and the imaginary dispersion curves with both $\omega / c$ and $k_{z}$ imaginary are located in 
separate sides of the asymptotic line $\beta_{a s y}=1 / \sqrt{\varepsilon_{r} \mu_{r}}$, and they never intersect. When an equiphase velocity line of $|\omega / c| /\left|k_{z}\right|=\beta_{\text {ph }}$ lies in the region $0<\beta_{\text {ph }}<\beta_{\text {asy }}$, all the modes corresponding to the intersections of the line with all the dispersion curves have imaginary eigenvalues, as shown in Fig. 1, while all the modes have real eigenvalues when $\beta_{\mathrm{ph}}>\beta_{\text {asy }}$. From this, we may conclude that all equiphase velocity modes either are real or are imaginary in a single-zone dielectric-filled waveguide.

One might conjecture that there would be no imaginary modes in the DLW accelerator structure, if both the loaded dielectric constant and the mode phase velocity are large enough, in other words, there would be no decaying modes if the particle energy is high enough so that $\beta^{2} \mu_{r} \varepsilon_{r}-1>$ 0 in the dielectric zone, with $\beta$ the drive particle velocity normalized to $c$. This is not true. To illustrate this, examples will be given below for two-zone structures; this structure is not suggested for any particular application, but is chosen only to simplify the analysis while retaining the essential physics. Numerical results for these illustrations were obtained from evaluations of dispersion equations that are derived in the next section.

For a multilayer DLW structure with a vacuum gap, imaginary dispersion curves may intersect the real dispersion curves, tending to go parallel to the light speed line when $\left|k_{z}\right|$ is large enough, as shown in Fig. 2 for $\mathrm{LSM}_{11}$ modes in a two-zone structure [14]. Consequently, a complete system of eigenmodes with $\beta_{\mathrm{ph}}<1$ in such a structure must include imaginary modes, no matter how small the gap width and how close to unity is the normalized

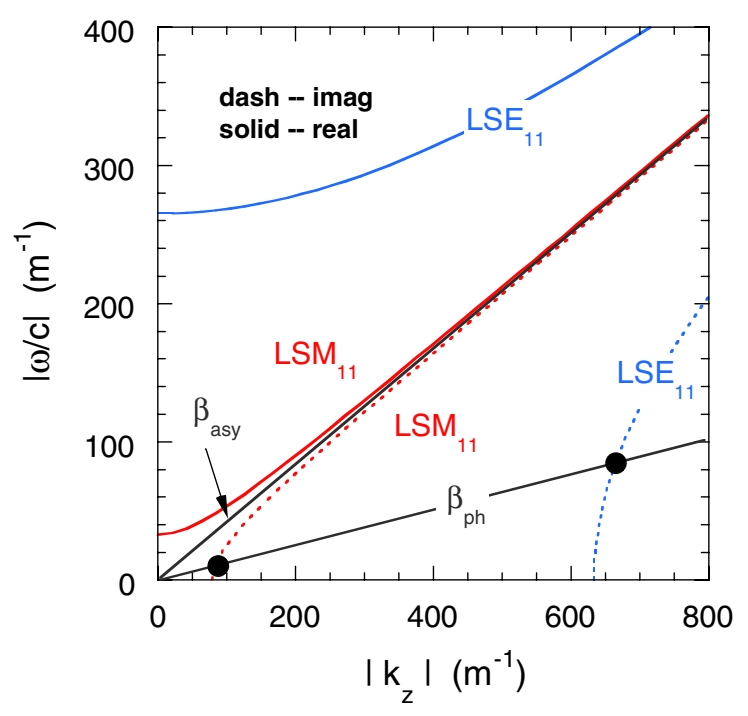

FIG. 1. (Color) Real and imaginary $\mathrm{LSM}_{11}$ - and $\mathrm{LSE}_{11}$-mode dispersion curves for a single-zone dielectric-filled rectangular waveguide, of which the width is $5 \mathrm{~mm}$ and the height is $40 \mathrm{~mm}$, with $\varepsilon_{r}=5.7$ and $\mu_{r}=1$. It is seen that the real and imaginary dispersion curves are located in separate sides of the asymptotic line $\beta_{\text {asy. }}$. The dots on the equiphase velocity line $\beta_{\mathrm{ph}}$ correspond to modes with imaginary eigenvalues. phase velocity $\beta_{\mathrm{ph}}$. In other words, decaying modes always exist in the field solution for DLW accelerator structures, for every value of drive particle energy. The decaying field is the particle's self-field, with a decay distance in the direction of motion that decreases as the particle velocity increases; this is the counterpart in a waveguide of the familiar contraction of fields into a "pancake," squeezing towards the plane of a relativistic particle. Figure 3 shows the dependence of decay length $\lambda_{d}$ on relativistic factor $\gamma_{\mathrm{ph}}$
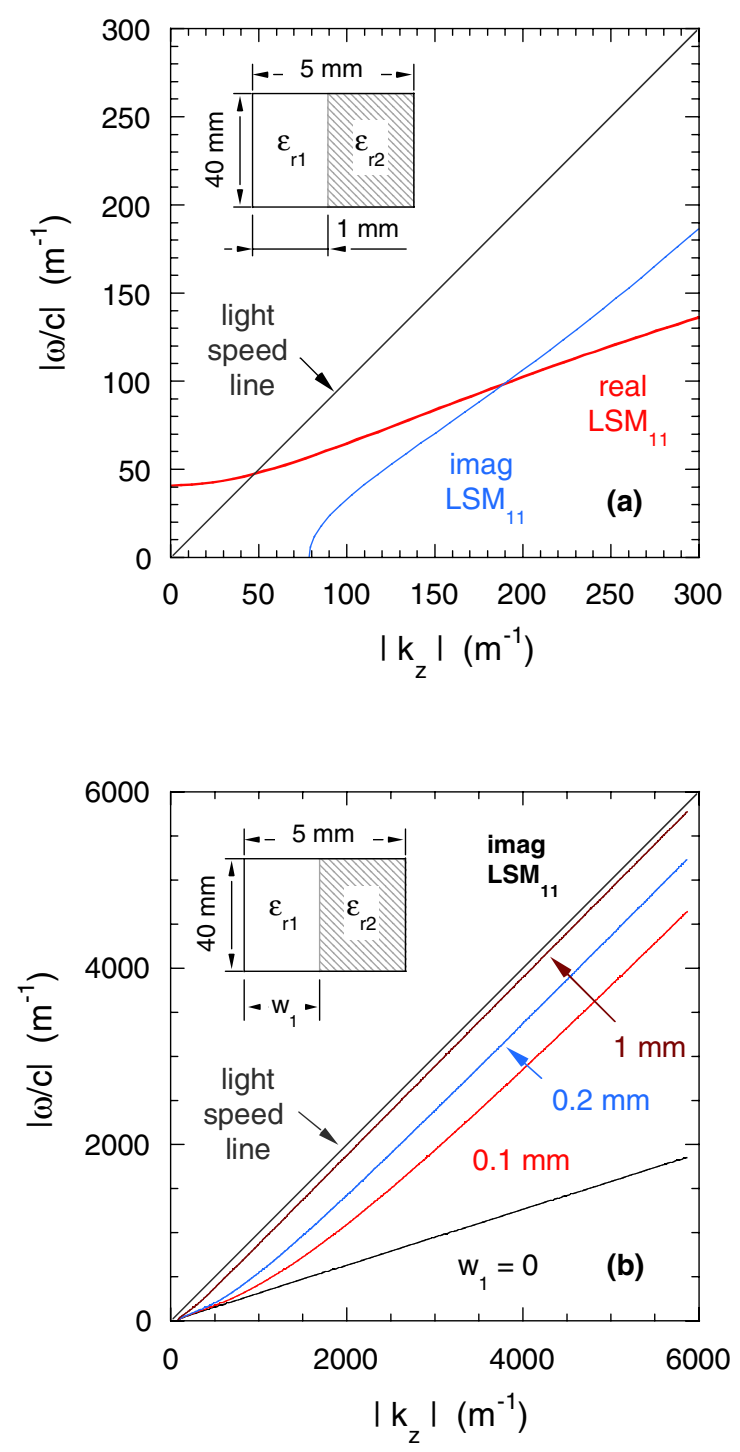

FIG. 2. (Color) (a) Both real and imaginary $\mathrm{LSM}_{11}$ mode dispersion curves for a vacuum gap of $w_{1}=1 \mathrm{~mm}$ in a two-zone rectangular DLW structure, with a width of $5 \mathrm{~mm}$ and a height of $40 \mathrm{~mm}$, with $\varepsilon_{r 1}=1, \varepsilon_{r 2}=10$, and $\mu_{r 1}=\mu_{r 2}=1$; (b) Only imaginary $\mathrm{LSM}_{11}$-mode dispersion curves for various vacuum gap widths $w_{1}$. It is seen from (a) that the imaginary curve can intersect the real curve, and from (b) that the curves with $w_{1} \neq 0$ become parallel to the light speed line when $\left|k_{z}\right|$ is large enough, although the smaller the gap width, the larger is the modulus of $k_{z}$ before the dispersion curve becomes parallel to the light line. 


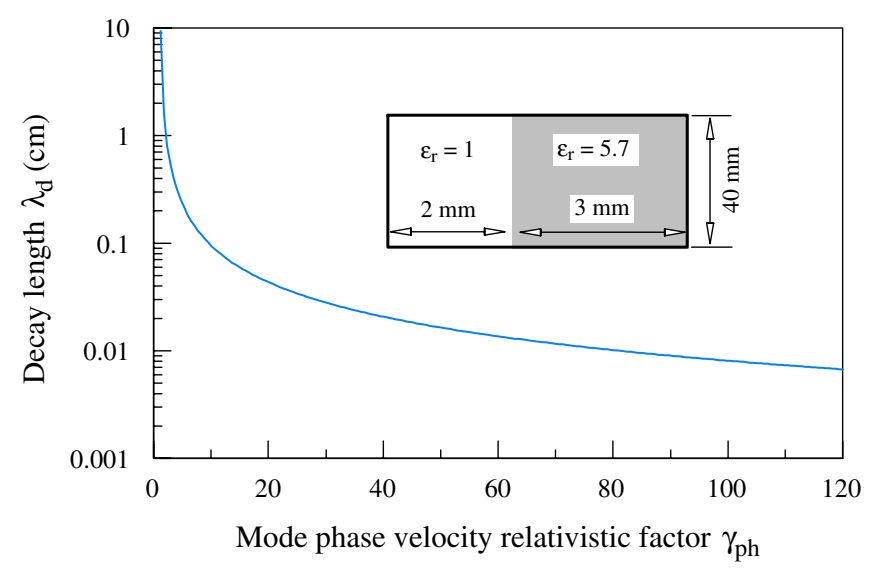

FIG. 3. (Color) Dependence of decay length $\lambda_{d}$ on relativistic factor $\gamma_{\mathrm{ph}}$ for imaginary mode $\mathrm{LSM}_{11}$ in a two-zone rectangular DLW structure, of which the width is $5 \mathrm{~mm}$ and the height is $40 \mathrm{~mm}$, with $\varepsilon_{r 1}=1, \varepsilon_{r 2}=5.7$, and $\mu_{r 1}=\mu_{r 2}=1$.

for a decaying $\mathrm{LSM}_{11}$ mode in a two-zone rectangular DLW structure, where $\lambda_{d}=2 \pi /\left|k_{z}\right|$ and $\gamma_{\mathrm{ph}}=$ $\left(1-\beta_{p h}^{2}\right)^{-1 / 2}$. It is seen that the decay length $\lambda_{d}$ decreases as $\gamma_{\mathrm{ph}}$ increases. For example, $\lambda_{d}$ is $1.24 \mathrm{~cm}$ for $\gamma_{\mathrm{ph}}=$ 1.98 (corresponding to $0.5-\mathrm{MeV}$ particle energy), while $\lambda_{d}$ is only $0.08 \mathrm{~mm}$ for $\gamma_{\mathrm{ph}}=100$ (50.6-MeV particle energy). This non-negligible range for the self-fields of a bunch, especially at low energies, shows the need to include decay modes in calculations of bunch dynamics. Moreover, it is seen that a possible new method for computing self-fields is provided by the theory given here. Heretofore, reliance was placed on computational methods using PIC codes to include self-fields [15].

It is found in the verification of the field solution for DLW structures that a complete system of equiphase velocity eigenmodes must satisfy the following two identities:

$$
\begin{gathered}
\sum_{m, n} \Pi_{z m n}^{-1} \varepsilon E_{z m n}^{*}\left(\mathbf{r}_{0}\right) E_{z m n}(\mathbf{r})=\delta\left(\mathbf{r}-\mathbf{r}_{0}\right) \\
\sum_{m, n} \Pi_{z m n}^{-1} E_{z m n}^{*}\left(\mathbf{r}_{0}\right) H_{z m n}(\mathbf{r})=0
\end{gathered}
$$

where $\mathbf{r}_{0}$ and $\mathbf{r}$ are any two locations in the cross section excluding points on dielectric interfaces or conducting walls, and where $\Pi_{z m n}$ is given in Eq. (16). Later we can see that Eqs. (17) and (18) can be taken to be a generalization of the property of TE and TM modes in a customary waveguide.

The existence of equiphase velocity imaginary modes with a common phase velocity $\beta_{\mathrm{ph}}<1$ in DLW accelerator structures can also be understood by examining the behavior of Eq. (17) in the neighborhood of $\mathbf{r}_{0}$. Suppose that $\mathbf{r}_{0}$ in Eq. (17) is located in the vacuum channel $\left(\mu_{r}=\varepsilon_{r}=1\right)$. For any real-modes $\left(k_{z}^{2}>0\right), E_{z}$-components vary exponentially with $x$ in the vacuum zone (where $x$ is the direction perpendicular to the dielectric interfaces), since $k_{x}^{2}=-\left(1-\beta_{\mathrm{ph}}^{2} \mu_{r} \varepsilon_{r}\right) k_{z}^{2}-k_{y}^{2}<0$; and generally speaking, a linear combination of exponential functions of $x$ cannot converge to $\delta\left(x-x_{0}\right)$. Thus only $E_{z}$ components of imaginary modes $\left(k_{z}^{2}<0\right)$, which are made up of sine or cosine functions, can allow it converge to $\delta\left(\mathbf{r}-\mathbf{r}_{0}\right)$. From this, Eq. (17) is consistent with the computational results shown in Fig. 2, and leads to a more general conclusion that a complete system of equiphase velocity eigenmodes must include decaying modes in a DLW structure if $\beta_{\mathrm{ph}}^{2} \mu_{r} \varepsilon_{r}-$ $1<0$ holds at least in one zone of the DLW structure.

It can be shown by direct calculations that Eq. (17) holds for one-dielectric-filled waveguide structures. Apparently, Eq. (18) also holds in such structures, since all TE and TM modes constitute a complete system of eigenfunctions with either zero $E_{z}$ or $H_{z}$, respectively. Of course, the choice of an eigenmode system may not be unique, just like the choice of the base vectors of a coordinate system, but a physical result will not depend on the choice, provided that the mode system is complete. For example, we can take LSM (longitudinal section magnetic) and LSE (longitudinal section electric) modes [14] (instead of TM and TE modes) as an eigenfunction system for the one-dielectricfilled rectangular structure; however, any eigenmode of the LSM-LSE system can be expressed in terms of TE and TM modes with the same eigenvalue (vice versa), resulting in the same conclusion. Therefore, Eqs. (17) and (18) are a generalization from customary (one-dielectric-filled) waveguide systems to general multilayer DLW systems. A general mathematical proof of the two identities is beyond the scope of this paper; nevertheless, a computational check is given below.

Carrying out the integration of Eqs. (17) and (18) with respect to $\mathbf{r}_{0}$ on the waveguide cross section, we have

$$
\begin{gathered}
I_{E}(\mathbf{r}) \equiv \sum_{m, n}\left(\Pi_{z m n}^{-1} \iint_{S} \varepsilon E_{z m n}^{*} d S\right) E_{z m n}(\mathbf{r})=1, \\
I_{H}(\mathbf{r}) \equiv \sum_{m, n}\left(\Pi_{z m n}^{-1} \iint_{S} \varepsilon E_{z m n}^{*} d S\right) \sqrt{\frac{\mu_{0}}{\varepsilon_{0}}} H_{z m n}(\mathbf{r})=0 .
\end{gathered}
$$

The quantity $I_{E}(\mathbf{r})$ can be taken as a sum of the two parts $\left(I_{E}\right)_{\mathrm{Re}}$ and $\left(I_{E}\right)_{\mathrm{Im}}$, where $\left(I_{E}\right)_{\mathrm{Re}}$ comes from the real eigenmodes while $\left(I_{E}\right)_{\mathrm{Im}}$ comes from the imaginary eigenmodes, and similarly for $I_{H}(\mathbf{r})$. These quantities are shown in Figs. 4 and 5 for a rectangular two-zone DLW structure given in Fig. 3, in which one of the zones is vacuum. In the computations leading to Figs. 4 and $5,1 \leq m \leq 20$ and $1 \leq n \leq 30$ are taken; in other words, there are 20 real and 20 imaginary modes for each $n$ of LSM and LSE modes. (Note that LSE modes with $n=0$ do not make contributions because $E_{z m 0}=0$.) The common phase velocity is taken to be $\beta_{\mathrm{ph}}=0.8629$, corresponding to a $0.5-\mathrm{MeV}$ particle energy, to avoid possible numerical problems due to overlarge moduli of the imaginary eigenvalues. It is seen 

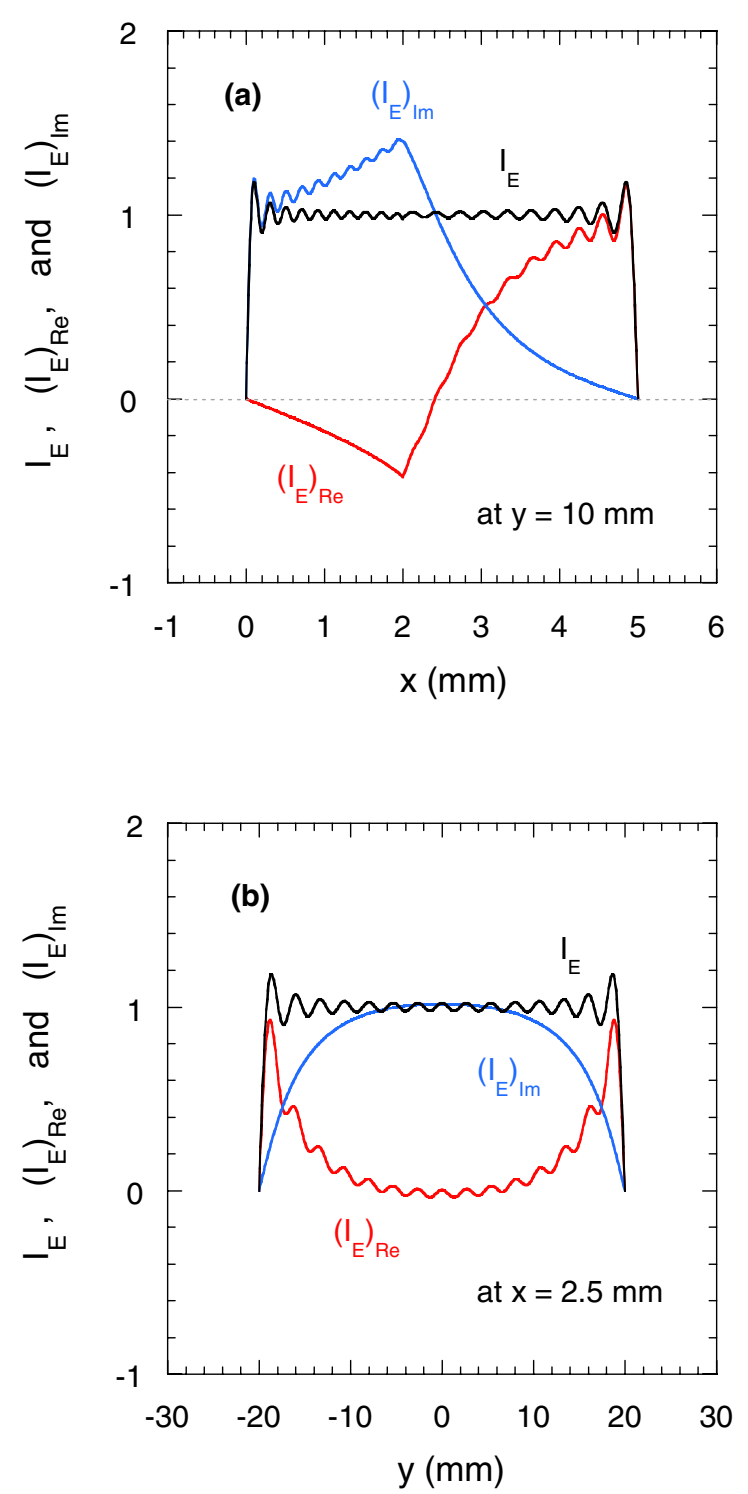

FIG. 4. (Color) Profiles of quantities $I_{E},\left(I_{E}\right)_{\mathrm{Re}}$, and $\left(I_{E}\right)_{\mathrm{Im}}$ for a rectangular two-zone DLW structure with a width of $5 \mathrm{~mm}$ and a height of $40 \mathrm{~mm}$. The first zone $(0<x<2 \mathrm{~mm})$ is a vacuum channel and the second zone $(2 \mathrm{~mm}<x<5 \mathrm{~mm})$ is a dielectric slab with $\varepsilon_{r 2}=5.7$ and $\mu_{r 2}=1$. (a) Profiles at $y=10 \mathrm{~mm}$; (b) profiles at $x=2.5 \mathrm{~mm}$. It is seen that $I_{E}$ ripples around unity and goes to zero rapidly on the waveguide walls $x=0$ and $5 \mathrm{~mm}$ in (a), and $y= \pm 20 \mathrm{~mm}$ in (b) required by boundary conditions.

from Figs. 4 and 5 that $I_{E}(\mathbf{r})$ converges reasonably to unity while $I_{H}(\mathbf{r})$ converges to zero. Clearly, the contribution from imaginary eigenmodes is essential in such a case; the set of real eigenmodes alone is not complete. Figure 6 shows two 3D plots for a direct demonstration of identities Eqs. (17) and (18), where $I_{E E}(x, y)=$ left-hand side of Eq. (17) and $I_{E H}(x, y)=$ left-hand side of Eq. (18) multiplied by $\varepsilon \sqrt{\mu_{0} / \varepsilon_{0}}$, both with $x_{0}=2.5 \mathrm{~mm}$ and $y_{0}=0$. In the computations, the mode index ranges are reduced for clarity from $(1 \leq m \leq 20,1 \leq n \leq 30)$ to $(1 \leq m, n \leq$ 10) to avoid too small mesh sizes. The $3 \mathrm{D}$ plots give a
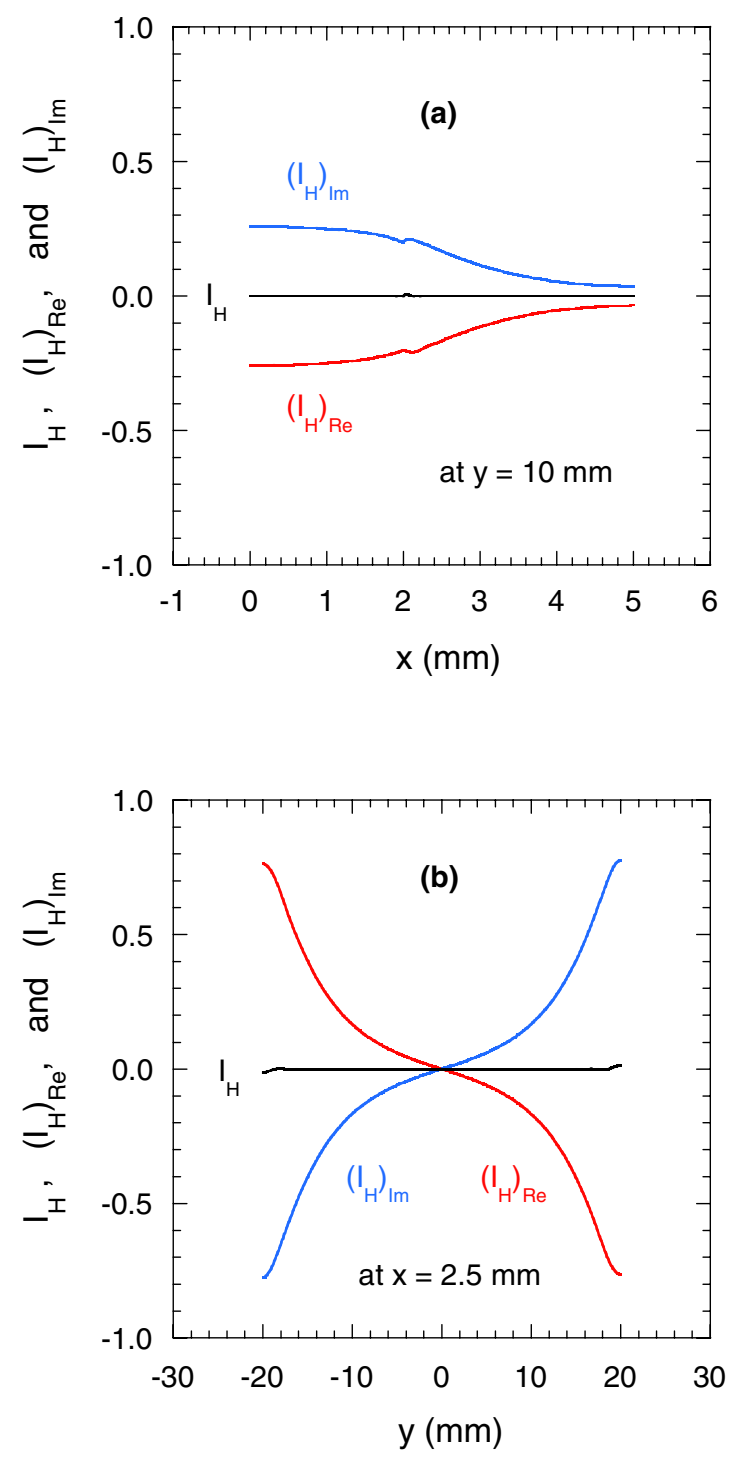

FIG. 5. (Color) Profiles of quantities $I_{H},\left(I_{H}\right)_{\mathrm{Re}}$, and $\left(I_{H}\right)_{\mathrm{Im}}$ for the two-zone DLW structure with parameters given in Fig. 4. (a) Profiles at $y=10 \mathrm{~mm}$; (b) profiles at $x=2.5 \mathrm{~mm}$. It is seen that the contributions from real and imaginary modes cancel each other so that $I_{H}$ well converges to zero.

clear general view although it is not convenient to get accurate readings in the figure.

In rectangular DLW structures, all LSM and LSE modes constitute a complete orthogonal system. Any field component of LSM modes $\left(H_{x}=0\right)$ can be expressed in terms of $E_{x}$, while any field component of LSE modes $\left(E_{x}=0\right)$ can be expressed in terms of $H_{x}$. In such a case, it is more convenient to use $E_{x}$ and $H_{x}$ to replace the orthogonality relation Eq. (14), to find

$$
\begin{aligned}
& \iint_{S}\left(v_{\mathrm{ph}}^{2} \mu \varepsilon-1\right) \varepsilon E_{x m n} E_{x p q}^{*} d x d y=\delta_{m n}^{p q} \Pi_{m n}^{(\mathrm{LSM})}, \\
& \iint_{S}\left(v_{\mathrm{ph}}^{2} \mu \varepsilon-1\right) \mu H_{x m n} H_{x p q}^{*} d x d y=\delta_{m n}^{p q} \Pi_{m n}^{(\mathrm{LSE})},
\end{aligned}
$$



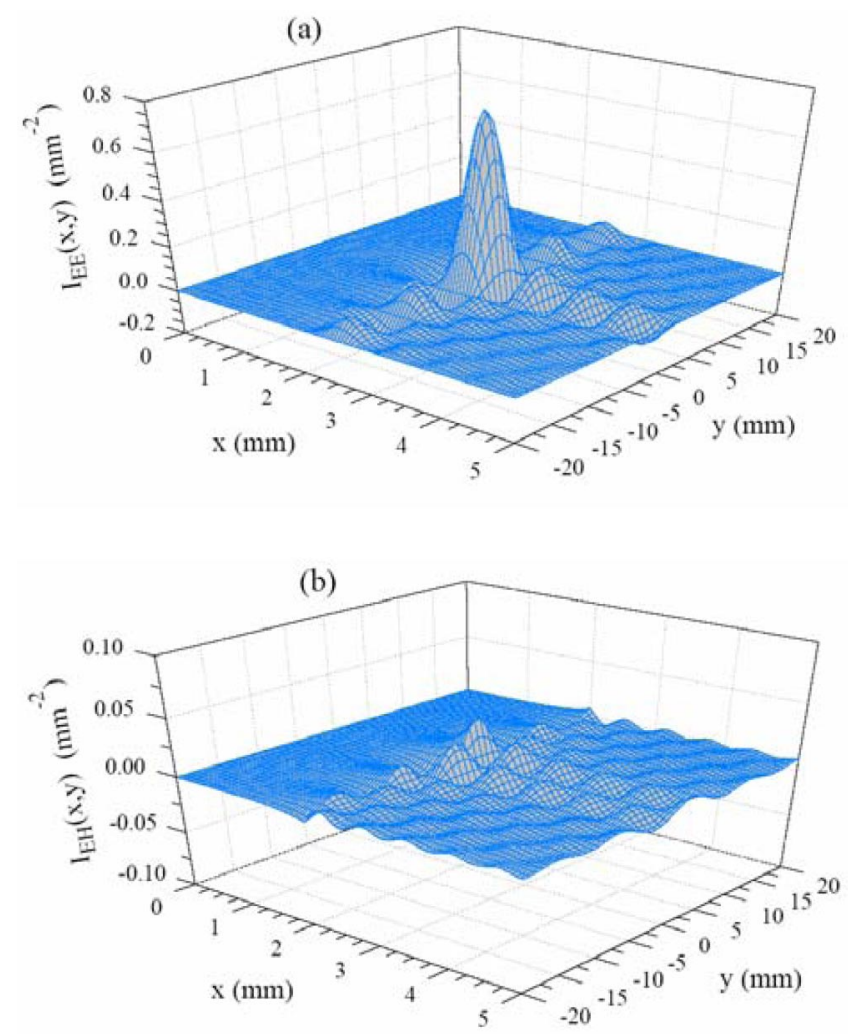

FIG. 6. (Color) Computational demonstration of Eqs. (17) and (18). (a) Dependence of $I_{E E}(x, y)$ and (b) dependence of $I_{E H}(x, y)$ on $x$ and $y$ on the cross section of the two-zone structure given in Fig. 4. It is seen that $I_{E E}(x, y)$ behaves as a $\delta$ function, while $I_{E H}(x, y)$ fluctuates around zero within $0.03 \mathrm{~mm}^{-2}$.

where $m$ and $n \geq 1$ for LSM modes, and $m \geq 1$ and $n \geq 0$ for LSE modes; $\Pi_{m n}^{(\mathrm{LSM})}$ and $\Pi_{m n}^{(\mathrm{LSE})}$ are self-defined by setting $p=m$ and $q=n$. The relations between $\Pi_{z m n}$ and $\Pi_{m n}^{(\mathrm{LSM})}$, and $\Pi_{m n}^{(\mathrm{LSE})}$ are given by

$$
\Pi_{z m n}=\frac{k_{z m n} k_{z m n}^{*}}{k_{y n}^{2}+k_{z m n}^{2}} \Pi_{m n}^{(\mathrm{LSM}, \mathrm{LSE})}
$$

where the mode labels LSM and LSE on $k_{z m n}$ and $\Pi_{z m n}$ are omitted, and $k_{y n}$ is the eigenvalue for the $y$ direction.

So far, the orthogonality conditions for equiphase velocity eigenmodes have been set up, the property of eigenvalues has been identified, and two basic identities have been confirmed by computations. Based on Eqs. (10) and (11), the differences between equiphase velocity and equifrequency modes in the DWL structure can be stated as follows: (1) The axial component product $\varepsilon k_{z m n} E_{z m n}\left(\omega_{p q}^{*} E_{z p q}^{*}\right)+\mu \omega_{m n} H_{z m n}\left(k_{z p q}^{*} H_{z p q}^{*}\right)$ is an orthogonal quantity for the equiphase velocity mode system, but it is not orthogonal for the traditional equifrequency mode system unless the structure is filled with only one dielectric. (2) The cross-product vector $\mathbf{E}_{\perp m n} \times \mathbf{H}_{\perp p q}^{*}$ is an orthogonal quantity for the equifrequency mode system, but it is not orthogonal for the equiphase velocity mode system unless the structure is filled with only one dielectric. (3) The phase velocity $v_{\mathrm{ph}}=\omega / k_{z}$ is real for the equiphase velocity mode system, while it is not necessarily true for the equifrequency mode system. (4) The equiphase velocity mode system is suitable for expansion of the electromagnetic fields which have a given phase velocity, such as the fields produced by a moving particle, while the equifrequency mode system is suitable for expansion of the electromagnetic fields which have a given frequency, such as the fields excited by an external rf source.

It should be noted that there is a limiting case not included in Eqs. (10) and (11), where the eigenmode system has a zero-phase velocity or zero frequency. In such a case, there is no coupling between $\mathbf{E}_{m n}(\mathbf{r})$ and $\mathbf{H}_{m n}(\mathbf{r})$ [see Eqs. (2)-(4)], that is, either $\mathbf{H}_{m n} \equiv 0$ or $\mathbf{E}_{m n} \equiv 0$ for the same mode; consequently, all the eigenmodes can be divided into two types: electric field modes and magnetic field modes. It can be shown that the orthogonality conditions in such a case are simplified to $\int \varepsilon E_{z m n} E_{z p q}^{*} d S=$ $\delta_{m n}^{p q} \Pi_{z m n}$ with $\Pi_{z m n}=\int \varepsilon\left|E_{z m n}\right|^{2} d S$ for the electric field modes $\left(\mathbf{H}_{m n} \equiv 0\right)$, and $\int \mu H_{z m n} H_{z p q}^{*} d S=\delta_{m n}^{p q} \Pi_{z m n}$ with $\Pi_{z m n}=\int \mu\left|H_{z m n}\right|^{2} d S$ for the magnetic field modes $\left(\mathbf{E}_{m n} \equiv 0\right)$; this is consistent with Eqs. (15) and (16). These eigenmodes can be used to expand the static fields produced by a charged particle at rest in a DLW structure.

\section{DISPERSION EQUATIONS}

Two-layer and symmetric three-layer rectangular DLW structures have been long studied $[14,16]$ and symmetric multilayer structures have been proposed for particle acceleration [8,17]. Recently, asymmetric structures were also suggested for particle acceleration in a two-beam scheme [10]. In this section, a general rectangular DLW structure with an arbitrary number of dielectric layers as shown in Fig. 7 will be analyzed, and dispersion equations for LSM and LSE modes will be derived.

For LSM modes $\left(H_{x}=0\right)$, the electric field component $E_{x}(x, y)$ perpendicular-to-dielectric interfaces is the generating component, through which all other components can be expressed, namely

$$
\begin{array}{cc}
E_{y}=\frac{1}{k_{y}^{2}+k_{z}^{2}} \frac{\partial^{2} E_{x}}{\partial x \partial y}, & E_{z}=\frac{-i k_{z}}{k_{y}^{2}+k_{z}^{2}} \frac{\partial E_{x}}{\partial x}, \\
H_{y}=\frac{\omega \varepsilon k_{z}}{k_{y}^{2}+k_{z}^{2}} E_{x}, & H_{z}=\frac{-i \omega \varepsilon}{k_{y}^{2}+k_{z}^{2}} \frac{\partial E_{x}}{\partial y} .
\end{array}
$$

The field expressions given in Eqs. (24) and (25) meet the first requirement on eigenmodes as mentioned previously: $\quad \mathbf{E}(x, y) \exp \left[i\left(\omega t-k_{z} z\right)\right]$ and $\quad \mathbf{H}(x, y) \times$ $\exp \left[i\left(\omega t-k_{z} z\right)\right]$ satisfy all source-free Maxwell equations. The second requirement that $\mathbf{E}(x, y)$ and $\mathbf{H}(x, y)$ satisfy all boundary conditions will be used to obtain dispersion equations. 


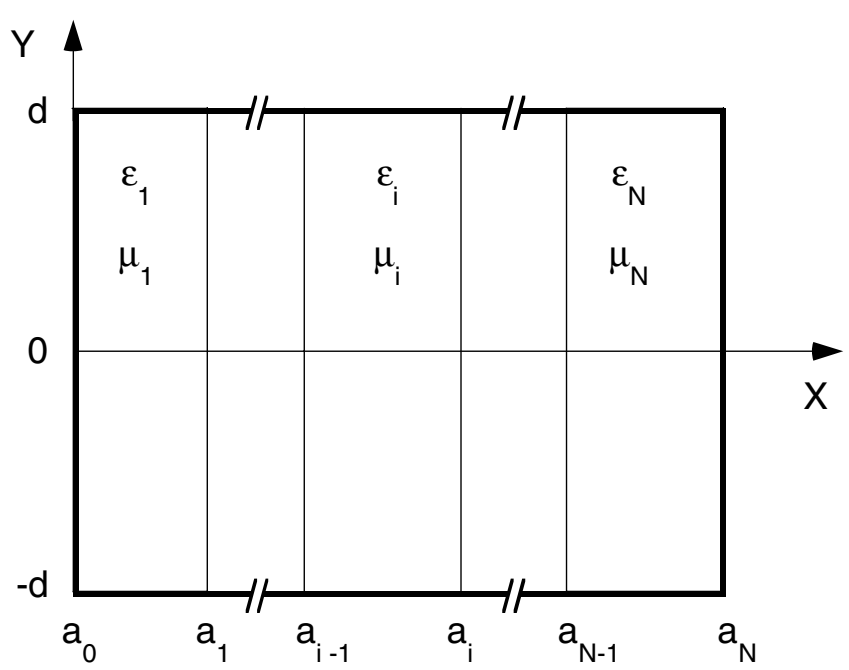

FIG. 7. Cross section of an $N$-zone dielectric-lined rectangular waveguide structure.

It can be generally shown (seen Appendix A) that the number of independent boundary conditions on individual dielectric interfaces for an eigenmode in the DLW structure is double the number of generating components. Since the LSM mode has just one generating component, only two of the five boundary conditions produced by five components are independent, which can be directly seen from Eqs. (24) and (25). If $E_{x}$ and $E_{z}$ satisfy all boundary conditions, then all the others automatically do. For the configuration shown in Fig. 7, the $E_{x}$ and $E_{z}$ components for the $i$ th zone can be written in the form of

$$
\begin{gathered}
E_{x}^{(i)}=e_{x}^{(i)}(x) \sin k_{y}(y+d), \\
E_{z}^{(i)}=-i e_{z}^{(i)}(x) \sin k_{y}(y+d),
\end{gathered}
$$

where

$$
\begin{aligned}
& e_{x}^{(i)}(x)=A^{(i)} \cos k_{x}^{(i)} x+B^{(i)} \sin k_{x}^{(i)} x, \\
& e_{z}^{(i)}(x)=\frac{k_{z}}{k_{y}^{2}+k_{z}^{2}} \frac{d e_{x}^{(i)}(x)}{d x},
\end{aligned}
$$

and $k_{y}=n \pi /(2 d)$ is the eigenvalue in the $y$ direction, $\omega^{2} \varepsilon_{i} \mu_{i}=\left(k_{x}^{(i)}\right)^{2}+k_{y}^{2}+k_{z}^{2}(i=1,2, \ldots, N)$, and $A_{i}$ and $B_{i}$ are constant coefficients. The dispersion equation as well as the $A_{i}$ and $B_{i}$ are to be determined from the boundary conditions for $\varepsilon_{i} e_{x}^{(i)}(x)$ and $d e_{x}^{(i)}(x) / d x$. From Eqs. (26) and (27), $\varepsilon_{i} e_{x}^{(i)}(x)$ and $d e_{x}^{(i)}(x) / d x$ can be written as

$$
\left(\begin{array}{c}
\varepsilon_{i} e_{x}^{(i)}(x) \\
\frac{d e_{x}^{(i)}(x)}{d x}
\end{array}\right)=M_{i}(x)\left(\begin{array}{c}
A^{(i)} \\
B^{(i)}
\end{array}\right)
$$

where

$$
M_{i}(x)=\left(\begin{array}{cc}
\varepsilon_{i} \cos k_{x}^{(i)} x & \varepsilon_{i} \sin k_{x}^{(i)} x \\
-k_{x}^{(i)} \sin k_{x}^{(i)} x & k_{x}^{(i)} \cos k_{x}^{(i)} x
\end{array}\right)
$$

The boundary conditions on $E_{x}$ and $E_{z}$ require that $\varepsilon_{i} e_{x}^{(i)}(x)$ and $d e_{x}^{(i)}(x) / d x$ be continuous at the dielectric interfaces $\left(x=a_{i}, i=1,2, \ldots, N-1\right)$, and $d e_{x}^{(i)}(x) / d x=$ 0 at the conducting walls ( $x=a_{0}$ and $a_{N}$ ). From Eq. (28) and the boundary conditions at $x=a_{i}, a_{i+1}, \ldots, a_{N-1}$, we obtain the recursion expression

$$
\left(\begin{array}{l}
A^{(i)} \\
B^{(i)}
\end{array}\right)=M_{i}^{-1}\left(a_{i}\right) S^{(i+1)} \cdots S^{(N-1)} M_{N}\left(a_{N-1}\right)\left(\begin{array}{c}
A^{(N)} \\
B^{(N)}
\end{array}\right),
$$

where

$$
\begin{aligned}
S^{(i)} & \equiv M_{i}\left(a_{i-1}\right) M_{i}^{-1}\left(a_{i}\right) \\
& =\left(\begin{array}{cc}
\cos \left(k_{x}^{(i)} w_{i}\right) & -\frac{\varepsilon_{i}}{k_{x}^{(i)}} \sin \left(k_{x}^{(i)} w_{i}\right) \\
\frac{k_{x}^{(i)}}{\varepsilon_{i}} \sin \left(k_{x}^{(i)} w_{i}\right) & \cos \left(k_{x}^{(i)} w_{i}\right)
\end{array}\right),
\end{aligned}
$$

with $w_{i}=a_{i}-a_{i-1}$ the $i$ th zone width.

From Eq. (28), the boundary conditions $d e_{x}^{(i)}(x) / d x=0$ at the conducting walls $\left(x=a_{0}\right.$ and $\left.a_{N}\right)$ can be written as

$$
\begin{aligned}
& {\left[\begin{array}{ll}
-\sin \left(k_{x}^{(1)} a_{0}\right) & \cos \left(k_{x}^{(1)} a_{0}\right)
\end{array}\right]\left(\begin{array}{l}
A^{(1)} \\
B^{(1)}
\end{array}\right)=0,} \\
& \left(\begin{array}{l}
A^{(N)} \\
B^{(N)}
\end{array}\right)=\left[\begin{array}{c}
\cos \left(k_{x}^{(N)} a_{N}\right) \\
\sin \left(k_{x}^{(N)} a_{N}\right)
\end{array}\right] \frac{A^{(N)}}{\cos \left(k_{x}^{(N)} a_{N}\right)} .
\end{aligned}
$$

Inserting Eq. (33) into Eq. (30) after setting $i=1$, and then inserting the resultant Eq. (30) into Eqs. (32), we obtain the LSM-mode dispersion equation

$$
\begin{aligned}
& \left(\begin{array}{ll}
\frac{k_{x}^{(1)}}{\varepsilon_{1}} \sin \left(k_{x}^{(1)} w_{1}\right) & \cos \left(k_{x}^{(1)} w_{1}\right)
\end{array}\right)\left(\prod_{j=2}^{N-1 \geq 2} S^{(j)}\right) \\
& \times\left(\begin{array}{c}
\cos \left(k_{x}^{(N)} w_{N}\right) \\
\frac{k_{x}^{(N)}}{\varepsilon_{N}} \sin \left(k_{x}^{(N)} w_{N}\right)
\end{array}\right)=0,
\end{aligned}
$$

where $\prod_{j=2}^{N-1 \geq 2} S^{(j)}=S^{(2)} S^{(3)} \cdots S^{(N-1)}$ with $N \geq 3$.

Without loss of generality, the zone number $N \geq 3$ was assumed for the sake of convenience in deriving the above dispersion equation. This dispersion equation is still applicable to one or two-zone structures $(N=1$ or 2$)$. This is because we can treat two zones as three zones by inserting an artificial boundary. Suppose that $x=a_{0}, a_{1}$, and $a_{3}$ are real boundaries for the two-zone structure while $a_{2}$ is the artificial one. Letting $a_{2}=a_{1}$, from Eq. (31) we find that $S^{(2)}$ is a unit matrix, and Eq. (34) is simplified to the twozone dispersion equation $\left(k_{x}^{(1)} / \varepsilon_{1}\right) \tan k_{x}^{(1)}\left(a_{1}-a_{0}\right)+$ $\left(k_{x}^{(3)} / \varepsilon_{3}\right) \tan k_{x}^{(3)}\left(a_{3}-a_{1}\right)=0$ [14], which was used for numerical examples in the last section. If the two zones 
have the same dielectric, the above equation is further simplified to $\sin k_{x}\left(a_{3}-a_{0}\right)=0$, the one-dielectric-filled dispersion equation.

From Eqs. (24)-(26), it is seen that all field components can be obtained from $e_{x}(x)$. Inserting Eq. (30) into Eq. (28), we obtain the $m$ - $n$ th--mode $e_{x}$ expression

$$
e_{x m n}^{(i)}(x)=\frac{1}{\varepsilon_{i}}\left(\cos k_{x m n}^{(i)}\left(a_{i}-x\right)-\frac{\varepsilon_{i}}{k_{x m n}^{(i)}} \sin k_{x m n}^{(i)}\left(a_{i}-x\right)\right) \xi_{m n}^{(i)},
$$

where

$$
\begin{aligned}
\xi_{m n}^{(i)} & =\left(\prod_{j=i+1}^{N-1} S_{m n}^{(j)}\right)\left(\begin{array}{c}
\cos \left(k_{x m n}^{(N)} w_{N}\right) \\
\frac{k_{x m n}^{(N)}}{\varepsilon_{N}} \sin \left(k_{x m n}^{(N)} w_{N}\right)
\end{array}\right) \frac{\varepsilon_{N} A_{m n}^{(N)}}{\cos k_{x m n}^{(N)} a_{N}}, \quad(1 \leq i \leq N-2), \\
\xi_{m n}^{(N-1)} & =\left(\begin{array}{c}
\cos \left(k_{x m n}^{(N)} w_{N}\right) \\
\frac{k_{x m n}^{(N)}}{\varepsilon_{N}} \sin \left(k_{x m n}^{(N)} w_{N}\right)
\end{array}\right) \frac{\varepsilon_{N} A_{m n}^{(N)}}{\cos k_{x m n}^{(N)} a_{N}}, \\
\xi_{m n}^{(N)} & =\left(\begin{array}{l}
1 \\
0
\end{array}\right) \frac{\varepsilon_{N} A_{m n}^{(N)}}{\cos k_{x m n}^{(N)} a_{N}} .
\end{aligned}
$$

It is seen from Eq. (36) that the eigenfields can differ by an arbitrary constant, and they are unique when normalized, for example, to the square root of $\Pi_{z m n}$ given in Eq. (23), which now can be rewritten as

$$
\begin{aligned}
\Pi_{z m n}= & \frac{k_{z m n} k_{z m n}^{*}}{k_{y n}^{2}+k_{z m n}^{2}} d \sum_{i=1}^{N}\left(v_{p h}^{2} \mu_{i} \varepsilon_{i}\right. \\
& -1) \varepsilon_{i} \int_{a_{i-1}}^{a_{i}}\left|e_{x m n}^{(i)}(x)\right|^{2} d x .
\end{aligned}
$$

For LSE modes $\left(E_{x}=0\right)$, the magnetic field component $H_{x}(x, y)$ is the generating component, but otherwise the treatment is similar. Below only the dispersion equation is given, written as

$$
\begin{aligned}
& \left(\cos \left(k_{x}^{(1)} w_{1}\right)-\frac{\mu_{1}}{k_{x}^{(1)}} \sin \left(k_{x}^{(1)} w_{1}\right)\right)\left(\prod_{j=2}^{N-1 \geq 2} S^{(j)}\right) \\
& \times\left(\begin{array}{c}
-\frac{\mu_{N}}{k_{x}^{(N)}} \sin \left(k_{x}^{(N)} w_{N}\right) \\
\cos \left(k_{x}^{(N)} w_{N}\right)
\end{array}\right)=0,
\end{aligned}
$$

where

$$
S^{(j)}=\left(\begin{array}{cc}
\cos \left(k_{x}^{(j)} w_{j}\right) & -\frac{\mu_{j}}{k_{x}^{(j)}} \sin \left(k_{x}^{(j)} w_{j}\right) \\
\frac{k_{x}^{(j)}}{\mu_{j}} \sin \left(k_{x}^{(j)} w_{j}\right) & \cos \left(k_{x}^{(j)} w_{j}\right)
\end{array}\right) .
$$

Note that in Eqs. (38) and (39), the same symbols $S^{(j)}$ and $k_{x}^{(j)}$ are used for simplicity.

\section{FIELD SOLUTION}

In this section, the eigenmode method presented previously will be employed to solve Maxwell equations for the electromagnetic field excited by a moving point charge in rectangular DLW structures. Then the point-charge solution, known as the Green's function, is directly used to obtain the solution for a continuous distribution of source (a finite bunch). The solution obtained here is applicable to general DLW structures, including the cylindrical one. Numerical examples are given to show that in the DLW structure with a vacuum zone, the field may include two parts: one is a radiation field or wakefield, while the other is a decaying field that decays away from the particle.

\section{A. Solution for a point charge}

Suppose that a particle having a charge of $e$ and initially located at $\left(x_{0}, y_{0}, z_{0}\right)$ moves at a constant velocity of $v$ along the $z$ direction. The charge and current density distributions are given by

$$
\rho=e \delta\left(x-x_{0}\right) \delta\left(y-y_{0}\right) \delta(s), \quad \mathbf{J}=\hat{\mathbf{z}} v \rho,
$$

where $s=v t-\left(z-z_{0}\right)$.

Usually, a standard procedure is used for solving electromagnetic problems in the waveguide systems: field generating components are obtained by solving wave equations, and the other components are obtained by using component-coupling equations; the procedure is similar to the one for establishing eigenmodes, as given in Eqs. (1)-(3), where $E_{z}$ and $H_{z}$ are taken as the generating components. For the field problem with a current density given in Eq. (40), it might appear to be more convenient to start from solving the $E_{z}$ - and $H_{z}$-wave equations, because $\mathbf{J}_{\perp}=0$ makes no contributions to the transverse field components and the component-coupling equations are greatly simplified. Unfortunately, there is a flaw in this procedure because, to employ the orthogonality given in Eq. (15), the axial electric and magnetic field components $E_{z}$ and $H_{z}$ have to be assumed to have the same expansion coefficients [2], which is not consistent with Maxwell curl equation $(\nabla \times \mathbf{E}=-\mu \partial \mathbf{H} / \partial t)$. To avoid this, a different procedure is used here: the transverse (perpendicular-to- 
dielectric interface) components $E_{x}$ and $H_{x}$ are taken as generating components while the other components are obtained from component-coupling equations. $E_{x}$ can be expanded only in terms of the LSM-mode eigenfunctions $\left\{E_{x m n}(x, y)\right\}$ while $H_{x}$ can be expanded only in terms of the LSE-mode eigenfunctions $\left\{H_{x m n}(x, y)\right\}$, because there is no coupling between $E_{x m n}$ and $H_{x m n}$ in the orthogonality relations given in Eqs. (21) and (22).

From Maxwell equations, the $E_{x}$ - and $H_{x}$-wave equations, and the component-coupling equations are given by

$$
\begin{gathered}
\left(\nabla^{2}-\varepsilon \mu \frac{\partial^{2}}{\partial t^{2}}\right) E_{x}^{(\mathrm{LSM})}=\frac{1}{\varepsilon} \frac{\partial \rho}{\partial x}+\mu \frac{\partial J_{x}}{\partial t}, \\
\left(E_{x}\right. \text {-wave equation), } \\
\left(\frac{\partial^{2}}{\partial x^{2}}-\varepsilon \mu \frac{\partial^{2}}{\partial t^{2}}\right) E_{y}=\frac{\partial^{2} E_{x}^{(L S M)}}{\partial y \partial x}-\mu \frac{\partial^{2} H_{x}^{(\mathrm{LSE})}}{\partial z \partial t}+\mu \frac{\partial J_{y}}{\partial t}, \\
\left(\frac{\partial^{2}}{\partial x^{2}}-\varepsilon \mu \frac{\partial^{2}}{\partial t^{2}}\right) E_{z}=\frac{\partial^{2} E_{x}^{(L S M)}}{\partial z \partial x}+\mu \frac{\partial^{2} H_{x}^{(\mathrm{LSE})}}{\partial y \partial t}+\mu \frac{\partial J_{z}}{\partial t}, \\
\left(\nabla^{2}-\varepsilon \mu \frac{\partial^{2}}{\partial t^{2}}\right) H_{x}^{(\mathrm{LSE})}=-\frac{\partial J_{z}}{\partial y}+\frac{\partial J_{y}}{\partial z}, \\
\left(\frac{\left.\partial^{2}-\mathrm{wave} \text { equation }\right),}{\partial x^{2}}-\varepsilon \mu \frac{\partial^{2}}{\partial t^{2}}\right) H_{y}=+\varepsilon \frac{\partial^{2} E_{x}^{(\mathrm{LSM})}}{\partial z \partial t}+\frac{\partial^{2} H_{x}^{(\mathrm{LSE})}}{\partial y \partial x}+\frac{\partial J_{z}}{\partial x}, \\
\left(\frac{\partial^{2}}{\partial x^{2}}-\varepsilon \mu \frac{\partial^{2}}{\partial t^{2}}\right) H_{z}=-\varepsilon \frac{\partial^{2} E_{x}^{(\mathrm{LSM})}}{\partial y \partial t}+\frac{\partial^{2} H_{x}^{(\mathrm{LSE})}}{\partial z \partial x}-\frac{\partial J_{y}}{\partial x},
\end{gathered}
$$

where $E_{x}^{(\mathrm{LSM})}$ denotes the $E_{x}$-component which can be expanded just in terms of $\left\{E_{x m n}\right\}$ while $H_{x}^{(\mathrm{LSE})}$ denotes the $H_{x}$ component which can be expanded just in terms of $\left\{H_{x m n}\right\}$.

The specific steps for the procedure are given as follows: (i) $E_{x}$ and $H_{x}$ are to be obtained by solving wave Eqs. (41) and (44). (ii) $E_{y}$ and $H_{z}$ are to be obtained from Eqs. (42) and (46), because $J_{y}=0$, and $E_{x}$ and $H_{x}$ are already known. Note that the derivatives $\partial / \partial z$ and $\partial / \partial t$ should be taken for two cases $(s>0$ and $s<0)$ because the contour integral for inverse Fourier transform is evaluated separately for the two cases. (iii) $E_{z}$ and $H_{y}$ are to be obtained from $\nabla \cdot \mathbf{E}=\rho / \varepsilon$ and $\nabla \cdot \mathbf{H}=0$, to avoid solving Eqs. (43) and (45) where $J_{z} \neq 0$. In calculating $E_{z}=$ $\int_{z}^{+\infty}\left(-\nabla_{\perp} \cdot \mathbf{E}_{\perp}+\rho / \varepsilon\right) d z$, the identity Eq. (17) and the $\delta$-function formula $\int_{z}^{+\infty} \delta(s) d z=\Theta(s)$ are needed, where $\Theta(s)$ is the unit step function; in calculating $H_{y}=$ $-\int_{y \text { on wall }}^{y}\left(\nabla_{\perp} \cdot \mathbf{H}_{\perp}\right) d y$, the starting point of $y$ is set on the conducting wall so that the boundary condition $H_{y}=0$ is satisfied.

From Eqs. (40), (41), and (44), the Fourier-transformed $E_{x^{-}}$and $H_{x}$-wave equations can be written as

$$
\left(\nabla_{\perp}^{2}+k_{\perp}^{2}\right)\left(\begin{array}{c}
\tilde{E}_{x}\left(x, y, k_{z}, \omega\right) \\
\tilde{H}_{x}\left(x, y, k_{z}, \omega\right)
\end{array}\right)=\left(\begin{array}{c}
\tilde{S}_{E} \\
\tilde{S}_{H}
\end{array}\right)
$$

where $\quad k_{\perp}^{2}=\omega^{2} \mu \varepsilon-k_{z}^{2}, \quad\left(\tilde{E}_{x}, \tilde{H}_{x}\right)=\int\left(E_{x}, H_{x}\right) \times$ $\exp \left[-i\left(\omega t-k_{z} z\right)\right] d z d t$, and

$$
\begin{aligned}
& \tilde{S}_{E}=\frac{2 \pi e}{\varepsilon} \delta^{\prime}\left(x-x_{0}\right) \delta\left(y-y_{0}\right) \delta\left(\omega-k_{z} v\right) e^{+i k_{z} z_{0}}, \\
& \tilde{S}_{H}=-2 \pi e v \delta\left(x-x_{0}\right) \delta^{\prime}\left(y-y_{0}\right) \delta\left(\omega-k_{z} v\right) e^{+i k_{z} z_{0}} .
\end{aligned}
$$

In Eq. (48), $\delta^{\prime}(\xi)$ denotes the derivative of delta function with respect to its argument. It is seen from above that the source terms $\tilde{S}_{E}$ and $\tilde{S}_{H}$ include a factor $\delta\left(\omega-k_{z} v\right)$, which means that all the fields produced by the particle have the same phase velocity $v$. It is the factor that ensures the applicability of the equiphase velocity eigenmode method.

Suppose that the $E_{x}$ and $H_{x}$ components expressed in terms of $\left\{E_{x m n}(x, y)\right\}$ and $\left\{H_{x m n}(x, y)\right\}$ are, respectively, given by

$$
\begin{aligned}
E_{x}^{(\mathrm{LSM})} & =\sum_{\mathrm{LSM}: m, n} \alpha_{x m n}(z, t) E_{x m n}(x, y), \\
H_{x}^{(\mathrm{LSE})} & =\sum_{\mathrm{LSE}: m, n} \beta_{x m n}(z, t) H_{x m n}(x, y) .
\end{aligned}
$$

Taking Fourier transform of Eq. (49) and inserting it into Eq. (47), we have

$$
\begin{gathered}
\sum_{\mathrm{LSM}: m, n}\left(k_{\perp}^{2}-k_{\perp m n}^{2}\right) \tilde{\alpha}_{x m n}\left(k_{z}, \omega\right) E_{x m n}(x, y)=\tilde{S}_{E}, \\
\sum_{\mathrm{LSE}: m, n}\left(k_{\perp}^{2}-k_{\perp m n}^{2}\right) \tilde{\beta}_{x m n}\left(k_{z}, \omega\right) H_{x m n}(x, y)=\tilde{S}_{H} .
\end{gathered}
$$

Although $\omega$ and $k_{z}$ are independent variables, $\omega / k_{z}$ in $k_{\perp}^{2}$ may be replaced by the particle velocity $v$ according to the $\delta$ function's selectivity; leading to $k_{\perp}^{2}=\left(v^{2} \mu \varepsilon-\right.$ 1) $k_{z}^{2}$. Then setting the eigenmode phase velocity $v_{p h}$ equal to $v$, we have $k_{\perp}^{2}-k_{\perp m n}^{2}=\left(v^{2} \mu \varepsilon-1\right)\left(k_{z}^{2}-k_{z m n}^{2}\right)$. Using the orthogonality Eqs. (21) and (22) in integrating the both sides of Eq. (50) over the cross section, we have 


$$
\begin{aligned}
\left(\begin{array}{c}
\tilde{\alpha}_{x m n}\left(k_{z}, \omega\right) \\
\tilde{\beta}_{x m n}\left(k_{z}, \omega\right)
\end{array}\right)= & \left(\begin{array}{c}
-\frac{e}{\Pi_{m n}^{(\mathrm{LSM})}} \frac{\partial E_{x m n}^{*}}{\partial x_{0}} \\
\frac{e v \mu}{\Pi_{m n}^{(\mathrm{LSE})}} \frac{\partial H_{x m n}^{*}}{\partial y_{0}}
\end{array}\right) \\
& \times \frac{2 \pi \delta\left(\omega-k_{z} v\right)}{\left(k_{z}^{2}-k_{z m n}^{2}\right)} e^{+i k_{z} z_{0}},
\end{aligned}
$$

where $E_{x m n}^{*}$ and $H_{x m n}^{*}$ take values at the particle location $\left(x_{0}, y_{0}\right)$.

Taking the inverse Fourier transform of Eq. (51) and inserting it into Eq. (49), we can get $E_{x}$ and $H_{x}$ solutions. The integration with respect to $\omega$ for the inverse transform is simple while the integration with respect to $k_{z}$ is a little tricky. The correct contours in the complex $k_{z}$ plane for the $k_{z}$ integration are shown in Fig. 8. It is seen that there are four possible poles. Two simple poles are shown on the real axis for $k_{z m n}^{2}>0\left(k_{z}= \pm k_{z m n}\right)$, which are responsible for propagating modes, and two are shown on the imaginary axis for $k_{z m n}^{2}<0\left(k_{z}= \pm i\left|k_{z m n}\right|\right)$, which are responsible for decaying modes. Evaluation of the $k_{z}$ integral for the inverse transform gives

$$
\int_{-\infty}^{+\infty} \frac{e^{i k_{z} s}}{\left(k_{z}^{2}-k_{z m n}^{2}\right)} d k_{z}=-\frac{2 \pi}{k_{z m n}} \psi_{\perp m n}(s)
$$

where

$$
\psi_{\perp m n}(s)= \begin{cases}\sin \left(k_{z m n} s\right) \Theta(s), & \left(k_{z m n}^{2}>0\right) \\ Z_{\perp m n}(s), & k_{z m n}^{2}<0\end{cases}
$$

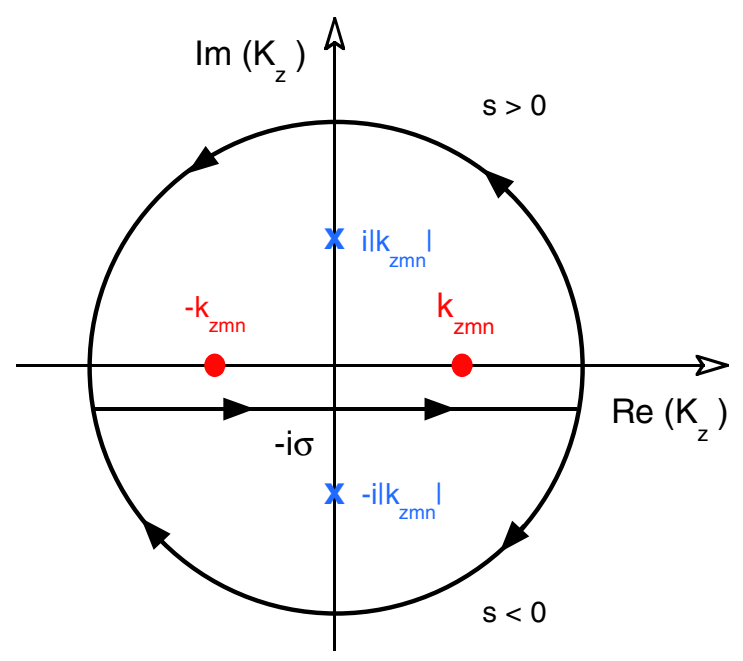

FIG. 8. (Color) Contours for integration in the $k_{z}$ plane. There are two kinds of possible poles: dots corresponding to propagating modes and crosses corresponding to decaying modes. The integral to be evaluated is defined on the line $\operatorname{Re}\left(k_{z}\right)-i \sigma$ in the lower-half plane, a little off the real axis, and its final result is obtained by letting $\sigma \rightarrow 0$ when the integration is finished. The contour closing in the upper-half plane is for $s=v t-(z-$ $\left.z_{0}\right)>0$, while the contour closing in the lower-half plane is for $s<0$. with

$$
\begin{aligned}
Z_{\perp m n}(s) & =\frac{1}{2 i}\left[e^{i k_{z m n} s} \Theta(s)+e^{-i k_{z m n} s} \Theta(-s)\right], \\
\left(k_{z m n}\right. & \left.=i\left|k_{z m n}\right|\right) .
\end{aligned}
$$

For the sake of definitude, $k_{z m n}=i\left|k_{z m n}\right|$ is defined for $k_{z m n}^{2}<0$ in Eq. (54) above.

Corresponding to $\psi_{\perp m n}(s)$ in Eq. (53), another useful function can be defined as

$$
\psi_{z m n}(s)= \begin{cases}\cos \left(k_{z m n} s\right) \Theta(s), & \left(k_{z m n}^{2}>0\right), \\ Z_{\| m n}(s), & \left(k_{z m n}^{2}<0\right),\end{cases}
$$

where

$$
\begin{aligned}
Z_{\| m n}(s) & =\frac{1}{2}\left[e^{i k_{z m n} s} \Theta(s)-e^{-i k_{z m n} s} \Theta(-s)\right], \\
\left(k_{z m n}\right. & \left.=i\left|k_{z m n}\right|\right) .
\end{aligned}
$$

Based on $E_{x}$ and $H_{x}$ components, all the other components can be obtained by following the steps (ii) and (iii) mentioned previously. With tedious but straightforward calculations omitted, the field solution for the moving point charge in the DLW structure can be written as the sum of all eigenmodes, given by

$$
\mathbf{E}_{\perp}=\sum_{m, n} \chi_{m n} \mathbf{E}_{\perp m n}(\mathbf{r}) \psi_{\perp m n}(s),
$$

$$
\begin{aligned}
& E_{z}=-\sum_{m, n} i \chi_{m n} E_{z m n}(\mathbf{r}) \psi_{z m n}(s), \\
& \mathbf{H}_{\perp}=\sum_{m, n} \chi_{m n} \mathbf{H}_{\perp m n}(\mathbf{r}) \psi_{\perp m n}(s), \\
& H_{z}=-\sum_{m, n} i \chi_{m n} H_{z m n}(\mathbf{r}) \psi_{z m n}(s),
\end{aligned}
$$

where all field components are real, although some eigenmodes may be imaginary, and the coefficient $\chi_{m n}$ can be expressed, whether for LSM or LSE modes, in the form of

$$
\chi_{m n}=-i e \Pi_{z m n}^{-1} E_{z m n}^{*}\left(\mathbf{r}_{0}\right) .
$$

In the above, the eigenmode symbols $\mathbf{E}_{m n}(x, y)$ and $\mathbf{H}_{m n}(x, y)$ are replaced by $\mathbf{E}_{m n}(\mathbf{r})$ and $\mathbf{H}_{m n}(\mathbf{r})$, because it can be shown (see Appendix B) that the solution is applicable to any longitudinally uniform DLW structures by just replacing the eigenmodes with those for the specific structures. This is reasonable if one considers that the expansion coefficients $\chi_{m n}, \psi_{\perp m n}(s)$, and $\psi_{z m n}(s)$ are only related to axial fields $E_{z}$ and $H_{z}$ at the particle location and axial eigenvalue $k_{z m n}$, independent of transverse geometry. The solution is also applicable to the limiting case where the point charge is at rest. In such a case, there are only decaying fields because all eigenvalues are imaginary, and there is no magnetic field because all the $\mathbf{H}_{m n}$ coefficients are equal to zero, resulting from the property of zero-phase velocity eigenmodes mentioned previously. 
It can be seen from the solution that the decaying fields characterized by the axial distribution functions $Z_{\perp m n}(s)$ and $Z_{\| m n}(s)$ decaying exponentially away from the particle $(s=0)$ are short-range self-fields, while the propagating radiation fields characterized by the trigonometric functions $\sin \left(k_{z m n} s\right)$ and $\cos \left(k_{z m n} s\right)$ are long-range radiation fields. Figure 9 is a numerical example showing the dependence of axial electric field $E_{z}$ on axial distance $z$ for propagating and decaying $\mathrm{LSM}_{11}$ modes excited by a $1-\mathrm{nC}$, $0.5 \mathrm{MeV}$ point bunch in a two-zone structure, with parameters as given in the figure caption. (Note the different scales for propagating and decay fields.) It is seen that the decaying mode is highly localized compared to the propagating mode.

Since the functions $\psi_{\perp m n}(s)$ and $\psi_{z m n}(s)$ are, respectively, included in the transverse and axial fields, it is informative to examine their behavior at the neighborhood of the particle. $\psi_{\perp m n}(s)$ is continuous at the particle location $(s=0)$, because

$$
\lim _{s \rightarrow 0_{-}} \psi_{\perp m n}(s)=\lim _{s \rightarrow 0_{+}} \psi_{\perp m n}(s)= \begin{cases}0, & \left(k_{z m n}^{2}>0\right), \\ -i / 2, & \left(k_{z m n}^{2}<0\right) .\end{cases}
$$

It is seen from above that the transverse electric fields are equal to zero for propagating modes $\left(k_{z m n}^{2}>0\right)$, which is consistent with the result given in Ref. [2], while there

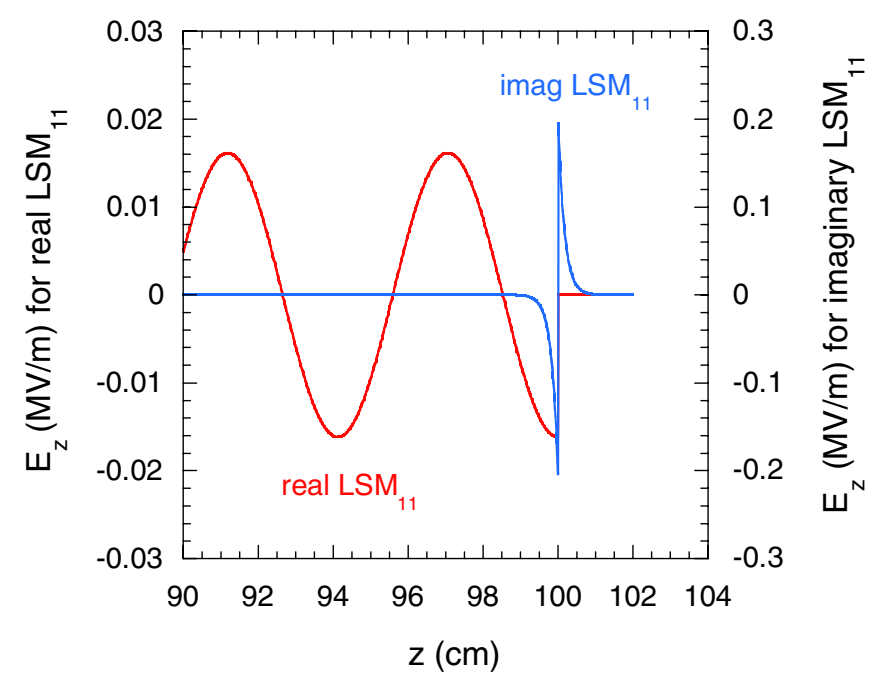

FIG. 9. (Color) Dependence of axial electric field $E_{z}$ on axial distance $z$ for propagating (real) and decaying (imaginary) $\mathrm{LSM}_{11}$ modes excited by a $1-\mathrm{nC}, 0.5-\mathrm{MeV}$ point bunch in a two-zone DLW structure with a height of $2 d=40 \mathrm{~mm}$, where the first zone $(0<x<2 \mathrm{~mm})$ is a vacuum channel and the second zone $(2 \mathrm{~mm}<x<5 \mathrm{~mm})$ is a dielectric slab with $\varepsilon_{r 2}=$ 5.7 and $\mu_{r 2}=1$. The bunch is located at the center of the vacuum channel $(x=1 \mathrm{~mm}$ and $y=0)$, and $z=100 \mathrm{~cm}$; the axial fields are plotted on the line which the bunch lies on. It is seen that the decaying mode is highly localized compared to the propagating mode that has a frequency of $4.4 \mathrm{GHz}$. Note the different scales for propagating and decay fields. are nonzero transverse electric fields for decaying modes $\left(k_{z m n}^{2}<0\right)$.

$\psi_{z m n}(s)$ is discontinuous at the particle location, because

$$
\lim _{s \rightarrow 0_{+}} \psi_{z m n}(s)= \begin{cases}1, & \left(k_{z m n}^{2}>0\right), \\ +1 / 2, & \left(k_{z m n}^{2}<0\right),\end{cases}
$$

(particle back face),

$$
\lim _{s \rightarrow 0_{-}} \psi_{z m n}(s)= \begin{cases}0, & \left(k_{z m n}^{2}>0\right), \\ -1 / 2, & \left(k_{z m n}^{2}<0\right),\end{cases}
$$

(particle front face),

where $\psi_{z m n}(0)=\frac{1}{2}$ for $k_{z m n}^{2}>0$ and $\psi_{z m n}(0)=0$ for $k_{z m n}^{2}<0$ can be defined according to the property of unit step function and $\delta$ function $\int_{-\infty}^{+\infty} \Theta(s) \delta(s) d s=\frac{1}{2}$. From this, the axial electric field $E_{z} \sim \psi_{z m n}(s)$ is not equal to zero at $s=0$ for propagating radiation modes, while it is equal to zero for decaying modes. That explains why the radiation modes take away energy from the particle while the decaying modes do not, if considering that the radiation power is evaluated from $\int\left(-J_{z} E_{z}\right) d V$ where $J_{z} \sim \delta(s)$ from Eq. (40). Since the radiation fields take away energy, the particle must experience a damping force or drag force [2], $F_{z}$, related to the radiation power $P_{\text {rad }}=$ $\int\left(-J_{z} E_{z}\right) d V=-v \int E_{z} \rho d V=-v F_{z}$.

It can be shown that the point-charge solution satisfies all Maxwell equations (see Appendix B). In a DLW accelerator structure, as mentioned previously, a complete solution must include decaying modes, and only propagating modes cannot exist. For example, electric force lines will stop at the $z$ plane on which the particle lies instead of at the charge, if only the propagating modes are counted in [2]. To satisfy Gauss's law $\nabla \cdot \mathbf{E}=\rho / \varepsilon$, the decaying modes are required.

To show the connection between propagating and decaying modes, a numerical example is given in Figs. 10 and 11 where electric force lines are drawn around a 1-nC, 0.5$\mathrm{MeV}$ point bunch on the $x-z$ symmetric plane $(y=0)$ in the structure given in Fig. 9. The force lines are in two dimensions on this plane, since the total $E_{y}$ is equal to zero although it may not for single modes. In Fig. 10, only 400 propagating modes $(m \times n=20 \times 10,200$ LSM modes and 200 LSE modes) are included. It is seen that electric force lines stop on the plane $s=0$ and there is no field in front of the bunch. In Fig. 11, all 400 propagating plus 400 decaying modes are included; in contrast to Fig. 10, all force lines stop on the bunch and the conducting walls, and in front of the bunch there are electric fields. (Note that the field strength is comparable by the line number per unit length across the lines only when following the line direction, while the strength is not comparable when along the direction perpendicular to the lines, because the lines are obtained by solving differential equations, instead of equiquantity line equations.) Obviously, Fig. 11 is consistent with Gauss law while Fig. 10 is not, which gives a direct 


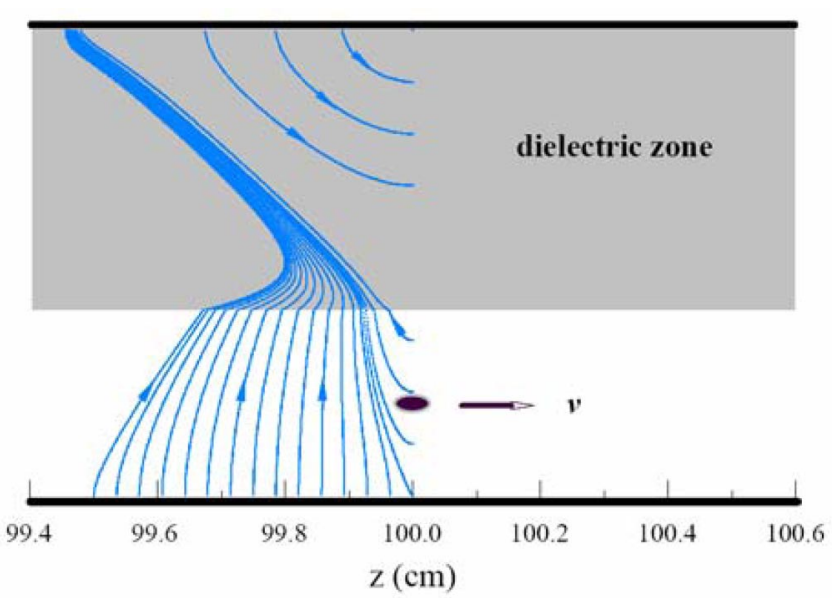

FIG. 10. (Color) Electric field force lines for 400 propagating modes around a moving point bunch in a DLW structure. Parameters for the structure and the bunch are given in Fig. 9. It is seen that there is no field in front of the bunch, and some force lines stop on the plane where the bunch is located, but not on the bunch.

illustration that propagating modes alone cannot exist in the DLW structure with a vacuum zone. By comparison, Fig. 12 shows a numerical example where the waveguide structure given in Fig. 10 is fully filled with one dielectric. In such a case, all modes are radiation modes, all force lines originate on the point bunch, and there is no field in front of the bunch.

Physically, when the energy velocities of propagating modes are less than the particle velocity, the particle loses energy, forming radiation. The radiation energy flows along but lags behind the particle. Inserting $E_{z}$ from Eq. (58) and $J_{z}$ from Eq. (40) into the formula $P_{\mathrm{rad}}=$

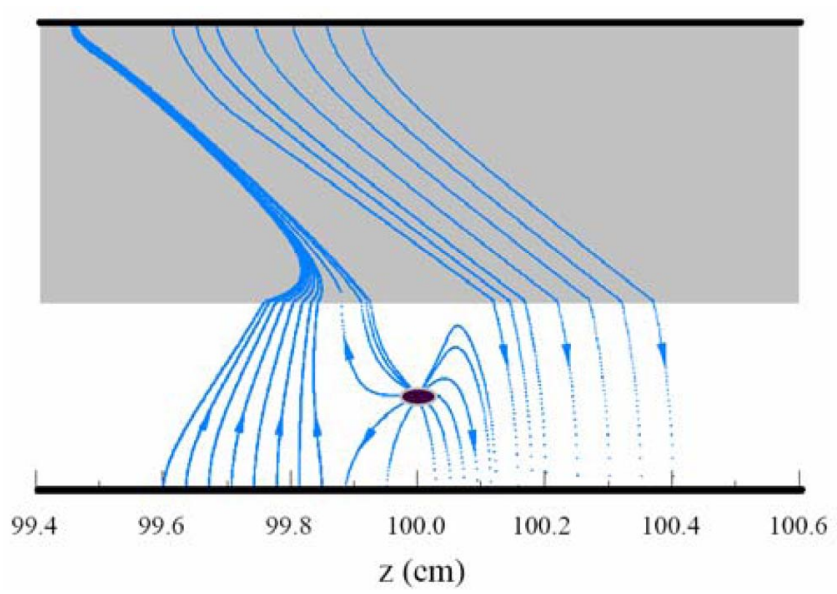

FIG. 11. (Color) Electric field force lines for 400 propagating and 400 decaying modes around a moving point bunch in a DLW structure. Parameters for the structure and the bunch are given in Fig. 9. Compared with Fig. 10 the essential difference is in that there are fields in front of the bunch and all force lines stop either on the bunch or on the conducting walls.

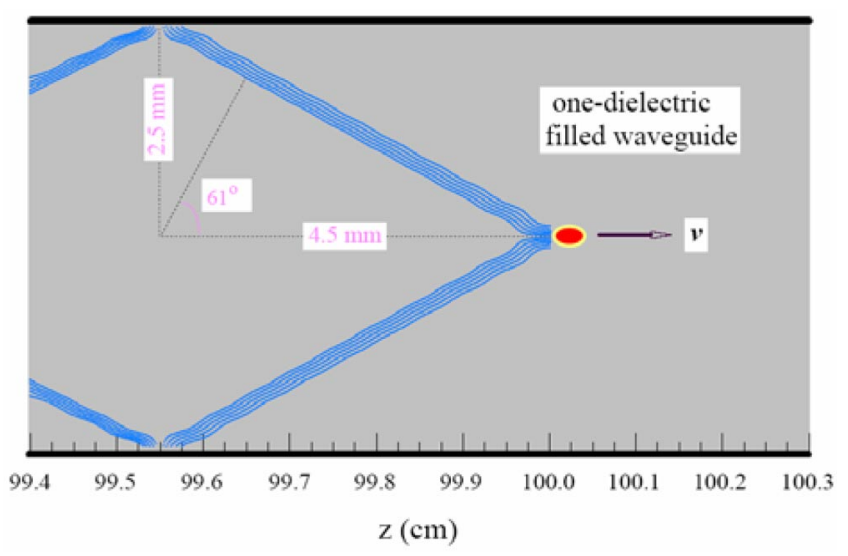

FIG. 12. (Color) Electric field force lines on the $x-z$ symmetric plane with respect to $y$, excited by a $0.5-\mathrm{MeV}, 1-\mathrm{nC}$ point bunch located at the cross section center ( $x=2.5 \mathrm{~mm}, y=0)$ and $z=$ $100 \mathrm{~cm}$ in the one-dielectric-filled rectangular waveguide with a width of $5 \mathrm{~mm}$ and a height of $40 \mathrm{~mm}$, and with $\varepsilon_{r}=5.7$ and $\mu_{r}=1$. In calculations, 400 (200 LSM and 200 LSE) modes are included. It is seen that the apparent Cerenkov angle $\tan ^{-1}(4.5 / 2.5)=60.95^{\circ}$ is in very good agreement with $60.96^{\circ}$ given by the classic formula $\cos ^{-1}\left(1 / \beta \sqrt{\varepsilon_{r} \mu_{r}}\right)$ with $\beta=$ 0.8629 . The finite thickness of the radiation cone is caused by numerical error since only a finite number of modes are included.

$\int\left(-J_{z} E_{z}\right) d V$, we can obtain the radiation power, which is applicable to any longitudinally uniform DLW structures, given by

$$
P_{\mathrm{rad}}=\sum_{m, n}^{\left(k_{z m n}^{2}>0\right)} P_{\mathrm{rad}}^{(m n)},
$$

where

$$
P_{\mathrm{rad}}^{(m n)}=\frac{1}{2} v e^{2} \frac{E_{z m n}^{*}\left(\mathbf{r}_{0}\right) E_{z m n}\left(\mathbf{r}_{0}\right)}{\prod_{z m n}} .
$$

The power given by Eq. (65) is positive definite, indicating the direction of power flow to be in the same direction as the particle motion; the claim in Ref. [2] that power flow was negative is seen to be erroneous. Note that the sum in Eq. (65) above is taken only over the propagating modes $\left(k_{z m n}^{2}>0\right)$; no time average is taken in obtaining Eq. (65), because different modes have different frequencies and time average is not applicable here. This means that the total radiation power is a constant (time-translation invariance). But the radiation power for a single mode varies with the time and the time average is applicable. $P_{\mathrm{rad}}^{(m n)}$ in Eq. (65) can be defined as the time-averaged power radiated into the $m-n$th mode by the particle. From the viewpoint of energy conservation, the radiation power $P_{\mathrm{rad}}^{(m n)}$ should be equal to the time-averaged flowing mode power $P_{\text {flow }}^{(m n)}$. Calculations show that $P_{\text {rad }}^{(m n)}$ is equal to $P_{\text {flow }}^{(m n)} \equiv$ $\left\langle\int\left(v^{2} \mu \varepsilon-1\right)\left(\mathbf{E}_{\perp}^{(m n)} \times \mathbf{H}_{\perp}^{(m n)}\right) \cdot \hat{\mathbf{z}} d S\right\rangle, \quad$ where $\quad \mathbf{E}_{\perp}^{(m n)}=$ 
$\chi_{m n} \mathbf{E}_{\perp m n}(\mathbf{r}) \sin \left(k_{z m n} s\right) \quad$ and $\quad \mathbf{H}_{\perp}^{(m n)}=\chi_{m n} \mathbf{H}_{\perp m n}(\mathbf{r}) \times$ $\sin \left(k_{z m n} s\right)$ are the $m$ - $n$ th-mode radiation fields given in Eqs. (57) and (59). From this, we find that the definition of radiation flowing power for individual modes excited by a drive particle is different from that of mode power excited by an external rf source [18]. It is interesting to indicate that if all modes are propagating modes, the total radiation power for a point charge would be infinite, namely $P_{\text {rad }}=$ $\left(v e^{2} / 2 \varepsilon\right) \lim _{\mathbf{r} \rightarrow \mathbf{r}_{0}} \delta\left(\mathbf{r}-\mathbf{r}_{0}\right)$ resulting from Eqs. (17) and (65); this is the case for Cerenkov radiation in an unbounded system as well, and originates from the assumption that $\varepsilon$ remains constant at high frequencies, clearly an unphysical assumption.

\section{B. Solution for a rigid finite-size bunch}

The solution for a rigid finite-size bunch can be obtained based on the point-charge solution. Suppose that the charge density distribution for a rigid bunch is described by $\rho_{b}(\mathbf{r}, z-v t)$. The solution for the bunch can be written as

$$
(\mathbf{E}, \mathbf{H})=\iiint \frac{\rho_{b}\left(\mathbf{r}_{0}, z_{0}\right)}{e}\left(\mathbf{E}_{G}, \mathbf{H}_{G}\right) d^{2} \mathbf{r}_{0} d z_{0},
$$

where $\left(\mathbf{E}_{G}, \mathbf{H}_{G}\right)$ denotes the point-charge solution, namely, the Green's function given in Eqs. (57)-(60), and $d^{2} \mathbf{r}_{0}$ denotes the differential area element in the transverse cross section. As a simple but typical example, the solution for a uniform bunch is given below.

Suppose that the charge density distribution of the uniform drive bunch is given by

$$
\rho_{b}(\mathbf{r}, z-v t)=\sigma_{d} \eta_{\perp}(\mathbf{r}) \eta_{z}(z-v t),
$$

where $\sigma_{d}$ is the constant density magnitude, $\eta_{\perp}(\mathbf{r})$ and $\eta_{z}(z-v t)$ are the transverse and axial distribution factors. The two factors are, respectively, given by

$$
\begin{aligned}
& \eta_{\perp}(\mathbf{r})= \begin{cases}1, & \text { within bunch, } \\
0, & \text { outside bunch, }\end{cases} \\
& \eta_{z}(z-v t)=\Theta\left(s_{b 2}\right)-\Theta\left(s_{b 1}\right),
\end{aligned}
$$

where $s_{b 1}=v t-\left(z-z_{b 1}\right)$ and $s_{b 2}=v t-\left(z-z_{b 2}\right)$, with $\Delta z_{b}=z_{b 2}-z_{b 1}$ the bunch length.

Inserting Eq. (67) into Eq. (66), we obtain the solution for a single uniform bunch moving through the longitudinally translationally invariant DLW structure, given by

$$
\begin{aligned}
\left(\begin{array}{c}
\mathbf{E}_{\perp} \\
\mathbf{H}_{\perp}
\end{array}\right)= & \sum_{m, n} \chi_{b m n}\left(\begin{array}{c}
\mathbf{E}_{\perp m n}(\mathbf{r}) \\
\mathbf{H}_{\perp m n}(\mathbf{r})
\end{array}\right)\left[\Theta\left(s_{b 2}\right)-\Theta\left(s_{b 1}\right)\right] \\
& -\sum_{m, n} \chi_{b m n}\left(\begin{array}{c}
\mathbf{E}_{\perp m n}(\mathbf{r}) \\
\mathbf{H}_{\perp m n}(\mathbf{r})
\end{array}\right)\left[\psi_{z m n}\left(s_{b 2}\right)-\psi_{z m n}\left(s_{b 1}\right)\right],
\end{aligned}
$$

$$
\begin{aligned}
\left(\begin{array}{c}
E_{z} \\
H_{z}
\end{array}\right)= & \sum_{m, n}-i \chi_{b m n}\left(\begin{array}{c}
E_{z m n}(\mathbf{r}) \\
H_{z m n}(\mathbf{r})
\end{array}\right)\left[\psi_{\perp m n}\left(s_{b 2}\right)\right. \\
& \left.-\psi_{\perp m n}\left(s_{b 1}\right)\right],
\end{aligned}
$$

where

$$
\chi_{b m n}=\frac{-i \sigma_{d}}{\Pi_{z m n} k_{z m n}} \iint \eta_{\perp}\left(\mathbf{r}_{0}\right) E_{z m n}^{*}\left(\mathbf{r}_{0}\right) d^{2} \mathbf{r}_{0} .
$$

The solution for a train of identical periodical bunches is the sum of individual bunches which are located at different axial locations. Suppose that the bunch number is $N_{b}$ and the bunch space period is $L_{b}$. The radiation power is given by

$$
P_{\mathrm{rad}}=\frac{1}{2} v \sigma_{d}^{2}\left(\Delta z_{b}\right)^{2} \sum_{m, n}^{\left(k_{z m n}^{2}>0\right)} C_{m n} \frac{\sin ^{2} \tau_{m n}}{\tau_{m n}^{2}} \frac{\sin ^{2}\left(N_{b} \zeta_{m n}\right)}{\sin ^{2} \zeta_{m n}},
$$

where

$$
\begin{aligned}
C_{m n} & =\Pi_{z m n}^{-1}\left|\iint \eta_{\perp}\left(\mathbf{r}_{0}\right) E_{z m n}^{*}\left(\mathbf{r}_{0}\right) d^{2} \mathbf{r}_{0}\right|^{2}, \\
\tau_{m n} & =\left(k_{z m n} \Delta z_{b}\right) / 2, \zeta_{m n}=\left(k_{z m n} L_{b}\right) / 2 .
\end{aligned}
$$

It is seen from Eq. (74) that $C_{m n}$ describes the interaction between mode fields and bunches, $\sin ^{2} \tau_{m n} / \tau_{m n}^{2}$ and $\sin ^{2}\left(N_{b} \zeta_{m n}\right) / \sin ^{2} \zeta_{m n}$ respectively describe the interfering effects from the bunch length $\Delta z_{b}$ and the bunch period $L_{b}$ $\left(>\Delta z_{b}\right)$. By setting $N_{b}=1, \sigma_{d}=e /\left(S_{b} \Delta z_{b}\right)$ with $S_{b}$ the bunch cross section area, and letting the bunch size go to zero, Eq. (73) is restored to Eq. (65) for the point-charge radiation power.

It is also seen from Eq. (73) that if the bunch period is set to be equal to the wavelength of the $m-n$th mode, the $m$ - $n$ th-mode radiation power is proportional to the square of the bunch number, and the power is strengthened coherently. If the power radiated by the first bunch is $P_{1}$, the power by the $N$ th bunch is $P_{N}=\left[N^{2}-(N-1)^{2}\right] P_{1}=$ $(2 N-1) P_{1}$ from energy conservation. A trailing bunch's radiation power is larger than a leading bunch's because it experiences a stronger deceleration force.

\section{CONCLUSIONS AND REMARKS}

In this paper, a formal eigenmode method in general translationally invariant DLW structures has been presented. This method has been employed to solve for fields excited by a moving charged particle in a rectangular DLW structure, and then the solution obtained is generalized to general DLW structures (including the cylindrical one). Numerical examples show that in a DLW structure that has a vacuum zone, the field solution may include both propagating modes and decaying modes; the propagating 
modes are long-range radiation fields or wakefields and the decaying modes are highly localized, short-range selffields.

Two important identities are found and confirmed by computations; the two identities are the theoretical basis of the eigenmode expansion technique. As is indicated in Appendix B, a field solution obtained through inverse Fourier transform may not satisfy Maxwell's equations at the singular point; therefore, verification of the solution becomes imperative. It is seen from Appendix B that the two identities play a key role in reaching the verification, although in the field solving process only identity Eq. (17) is used when calculating $E_{z}=\int\left(-\nabla_{\perp} \cdot \mathbf{E}_{\perp}+\rho / \varepsilon\right) d z$, where $\rho \sim \delta\left(\mathbf{r}-\mathbf{r}_{0}\right)$ needs to be expanded in terms of eigenfunction $\left\{E_{z m n}(\mathbf{r})\right\}$.

Mathematically, the convergence of an expansion in terms of eigenmodes satisfying the boundary conditions of DLW structures is a significant topic, which is beyond the scope of this paper. However, due to the existence and uniqueness of a solution to the physical problem itself, the solution expansion is supposed to be convergent. In numerical calculations, identity Eq. (19) resulting from Eq. (17) can be used for checking if any influential modes are lost in finding roots (eigenvalues) and provide a rule of thumb for determining how many modes are needed based on required precisions.

According to the relativity principle, all the electric force lines produced by a charged particle moving at the light speed in free space stop at the particle, concentrating in the plane fixed to the particle and perpendicular to the direction of motion; the transverse electric fields become infinite while the axial electric field vanishes. After Ng's work [3], Burov and Novokhatski evidently realized that there should be decaying fields and electric force lines stopping on a charged bunch when the bunch passes though a vacuum pipe in a cylindrical dielectric-lined waveguide [19]. In their calculations, the authors assumed that the bunch moves at the vacuum light speed, but they found a finite imaginary axial wave number for $k_{z}$, which is not consistent with the relativity principle, because $\left|k_{z}\right|$ in such a case should go to infinity and the electric force lines in the vacuum pipe should stop vertically at the bunch.

The point-charge solution (Green's function) is a basic technique in electromagnetic theory. As mentioned previously, there are two methods often used for solving boundary-value problems: the eigenmode method presented in this paper and the superposition method used in some other publications [3,4]. From Appendix C, we know that the superposition method is based on a linear combination of a particular solution and a general solution. It should be noted that the general and particular solutions satisfy the same equation except for at the singular point. That means that each zone in the DLW structure must correspond to its own particular and general solutions because the wave equation depends on dielectric parame- ters; in other words, the solution in a specific zone should be the combination of its own particular and general solutions. But it is not necessary to do that to simplify calculations for some specific situations. For example, in the calculation of the two-zone cylindrical DLW accelerator structure $[3,4]$, only one particular solution that corresponds to the inner vacuum zone is used to match boundary conditions while the particular solution of the outer dielectric zone is taken to be zero. Such a treatment makes sense physically because the particle is assumed only to move in the inner vacuum zone. Of course, this simplification comes at the expense of the universality of the solution obtained; for example, the solution is not applicable to the opposite case where the inner zone is dielectric while the outer zone is vacuum beam channel.

\section{ACKNOWLEDGMENTS}

Appreciation is extended to T.C. Marshall and V. P. Yakovlev for stimulating and helpful discussions and to V.P. Yakovlev for drawing our attention to Ref. [19]. This work was supported by the U.S. Department of Energy, Office of High Energy Physics.

\section{APPENDIX A: BOUNDARY CONDITIONS}

In this appendix, it will be shown that only two boundary conditions for each generating component are independent on individual dielectric interfaces for an eigenmode in the translationally invariant DLW structures, while only one is independent on conducting walls. The conclusion is applicable to both equiphase velocity and equifrequency eigenmodes.

In the DLW structures, there are two kinds of physical boundaries: a conducting wall and a dielectric interface. In the separated-variable method for solving partial differential equations, the dielectric interfaces and conducting walls are supposed to coincide with the coordinate surfaces of a coordinate system to make boundary conditions employable. As a result, analytically solvable DLW structures are very limited. To judge the solvability of eigenvalue problems, it is helpful to know whether the number of all the solution-function coefficients in the structure is equal to the number of all the independent boundary conditions. Furthermore, it is also helpful to know what boundary conditions are independent and what are equivalent, so that a proper choice of the boundary conditions can be made to simplify calculations.

As it is well known, all field components of an eigenmode can be expressed in terms of one or at most two generating components. The choice of generating components is not unique; however, the choice of axial components as the generating components is universal. To show the above conclusion, we start from eigenmode basic equations. 
Suppose that $\hat{\mathbf{n}}$ and $\hat{\mathbf{t}}$ are, respectively, the normal and tangential unit vectors on the dielectric or conducting boundary in the $x-y$ plane, with $\hat{\mathbf{n}}=\hat{\mathbf{t}} \times \hat{\mathbf{z}}, \hat{\mathbf{t}}=\hat{\mathbf{z}} \times \hat{\mathbf{n}}$, and $\hat{\mathbf{z}}=\hat{\mathbf{n}} \times \hat{\mathbf{t}}$. Equation (2) $+\left(\mu \omega / k_{z}\right) \hat{\mathbf{z}} \times$ Eq. (3) and Eq. (3) $-\left(\varepsilon \omega / k_{z}\right) \hat{\mathbf{z}} \times$ Eq. (2) yield

$$
\begin{aligned}
& \mathbf{E}_{\perp}+v_{\mathrm{ph}} \hat{\mathbf{z}} \times \mu \mathbf{H}_{\perp}=i k_{z}^{-1} \nabla_{\perp} E_{z}, \\
& \mathbf{H}_{\perp}-v_{\mathrm{ph}} \hat{\mathbf{z}} \times \varepsilon \mathbf{E}_{\perp}=i k_{z}^{-1} \nabla_{\perp} H_{z},
\end{aligned}
$$

where the mode indices $m n$ are omitted.

On the dielectric interface, six boundary conditions produced by six field components require that $\left(\varepsilon E_{n} ; E_{t}, E_{z}\right)$ and $\left(\mu H_{n} ; H_{t}, H_{z}\right)$ be continuous. Dotmultiplying both sides of Eqs. (A1) and (A2) by $\hat{\mathbf{t}}$, we have

$$
\begin{aligned}
& E_{t}+v_{\mathrm{ph}} \mu H_{n}=i k_{z}^{-1}\left(\hat{\mathbf{t}} \cdot \nabla_{\perp} E_{z}\right), \\
& H_{t}-v_{\mathrm{ph}} \varepsilon E_{n}=i k_{z}^{-1}\left(\hat{\mathbf{t}} \cdot \nabla_{\perp} H_{z}\right),
\end{aligned}
$$

where the six components $\left(\varepsilon E_{n} ; E_{t}, E_{z}\right)$ and $\left(\mu H_{n} ; H_{t}, H_{z}\right)$ are all included.

On the conducting wall, the boundary conditions require that $\left(E_{t}, E_{z} ; H_{n}\right)$ be equal to zero. Dot-multiplying Eqs. (2) and (3) in the form of $\hat{\mathbf{t}} \cdot$ Eq. (2) and $\hat{\mathbf{n}} \cdot$ Eq. (3), we have

$$
\begin{aligned}
& E_{t}=-i k_{\perp}^{-2}\left[k_{z}\left(\hat{\mathbf{t}} \cdot \nabla_{\perp} E_{z}\right)-\omega \mu\left(\hat{\mathbf{n}} \cdot \nabla_{\perp} H_{z}\right)\right], \\
& H_{n}=-i k_{\perp}^{-2}\left[k_{z}\left(\hat{\mathbf{n}} \cdot \nabla_{\perp} H_{z}\right)-\omega \varepsilon\left(\hat{\mathbf{t}} \cdot \nabla_{\perp} E_{z}\right)\right],
\end{aligned}
$$

where the three components $\left(E_{t}, E_{z} ; H_{n}\right)$ are all included.

The proof given below includes two cases. (1) Two generating components: there are four independent boundary conditions on the dielectric interface and two on the conducting wall; (2) one generating component: there are two independent boundary conditions on the dielectric interface and one on the conducting wall.

(1) Two generating components $E_{z}$ and $H_{z}$ (hybrid electric and magnetic modes).

On dielectric boundaries. - There are only three terms in Eqs. (A3) and (A4), and if any two of the three terms are continuous, then the third term must be continuous. Consequently, only four boundary conditions are independent on the dielectric interface. Mathematically, the continuations of $E_{z}$ and $H_{z}$ result in the continuations of $\left(\hat{\mathbf{t}} \cdot \nabla_{\perp} E_{z}\right)$ and $\left(\hat{\mathbf{t}} \cdot \nabla_{\perp} H_{z}\right)$, respectively. Therefore, any two of $\left(\varepsilon E_{n} ; E_{t}, E_{z}\right)$ 's continuations plus any two of $\left(\mu H_{n} ; H_{t}, H_{z}\right)$ 's continuations make up a set of independent boundary conditions. The combination of $\left(E_{t}, E_{z}\right)$ and $\left(H_{t}, H_{z}\right)$ is one of the several choices, for example.

On conducting boundaries. - It is seen that Eqs. (A5) and (A6) are linear equations for the four quantities $E_{t},(\hat{\mathbf{t}}$. $\left.\nabla_{\perp} E_{z}\right), H_{n}$, and $\left(\hat{\mathbf{n}} \cdot \nabla_{\perp} H_{z}\right)$; if any two of the four are equal to zero, the other two must be equal to zero. From this, only two boundary conditions are independent. The choice of $E_{z}=0$ and $\left(\hat{\mathbf{n}} \cdot \nabla_{\perp} H_{z}\right)=0\left(\partial H_{z} / \partial n=0\right)$ is one of the several choices, for example. Note that $E_{z}=0$ instead of $\hat{\mathbf{t}} \cdot \nabla_{\perp} E_{z}=0$ is taken here. Mathematically, $E_{z}=0$ is a sufficient condition resulting in $\hat{\mathbf{t}} \cdot \nabla_{\perp} E_{z}=0$ but not a necessary one, and physically, $E_{z}=0$ is the original boundary condition.

(2) One generating component $E_{z}$ (TM modes) or $H_{z}$ (TE modes).

The proof for TE modes is similar to the proof for TM modes, because the component-coupling equations for TE modes can be obtained from the ones for TM mode just by making substitutions: $E \rightarrow H, H \rightarrow E, \varepsilon \rightarrow-\mu$, and $\mu \rightarrow-\varepsilon$. For this, only the proof for TM modes is given below.

On dielectric boundaries. - The boundary conditions require that $\left(\varepsilon E_{n} ; E_{t}, E_{z}\right)$ and $\left(\mu H_{n} ; H_{t}, H_{z} \equiv 0\right)$ be continuous on the dielectric interface. From Eqs. (2) and (A2), we have $\mu H_{n}=-\left(\omega \mu \varepsilon / k_{z}\right) E_{t}=i\left(\omega \mu \varepsilon / k_{\perp}^{2}\right)\left(\hat{\mathbf{t}} \cdot \nabla_{\perp} E_{z}\right)$. From this, it is seen that $\mu H_{n}$ and $E_{t}$ cannot satisfy boundary conditions simultaneously unless $\hat{\mathbf{t}} \cdot \nabla_{\perp} E_{z} \equiv 0$ for a DLW structure with different dielectrics. $\hat{\mathbf{t}} \cdot \nabla_{\perp} E_{z} \equiv$ 0 means that $E_{z}$ is not a function of the coordinate variable in the $\hat{\mathbf{t}}$ direction, which corresponds to $\mathrm{TM}_{0 n}$ modes in the cylindrical DLW structure, for example. Thus the boundary conditions become that $\left(\varepsilon E_{n} ; E_{t} \equiv 0, E_{z}\right)$ and $\left(\mu H_{n} \equiv\right.$ $\left.0 ; H_{t}, H_{z} \equiv 0\right)$ are continuous, with only three nonzero. Equation (A3) is automatically satisfied and Eq. (A4) becomes $H_{t}-v_{\mathrm{ph}} \varepsilon E_{n}=0$, which means that the continuities of $H_{t}$ and $\varepsilon E_{n}$ are equivalent, and thus the continuation of $E_{z}$ plus any one of $\left(\varepsilon E_{n} ; H_{t}\right)$ 's continuations makes up a set of independent boundary conditions. Therefore, only two boundary conditions are independent on the dielectric interface.

On conducting boundaries. - As mentioned above, the six components for TM modes are given by $\left(\varepsilon E_{n} ; E_{t} \equiv\right.$ $\left.0, E_{z}\right)$ and $\left(\mu H_{n} \equiv 0 ; H_{t}, H_{z} \equiv 0\right)$. The original boundary conditions on the conducting wall are $\left(E_{t}, E_{z} ; H_{n}\right)=0$. Since $E_{t} \equiv 0$ and $H_{n} \equiv 0$, the only boundary condition is $E_{z}=0$.

\section{APPENDIX B: VERIFICATION OF SOLUTION}

In this appendix, it will be generally shown that the point-charge solution given in Eqs. (57)-(60) satisfies all Maxwell equations; in other words, the solution is applicable to any longitudinally translationally invariant DLW structures.

It is important itself to prove that the solution obtained satisfies Maxwell's equations, especially at the singular point. The point-charge solution mathematically is a Green's function, and the singularity in the Green's function is artificially made up in a sense because there are different ways to construct the singularity. For example, the inverse Fourier transform used for solving wave equations is evaluated by carrying out contour integration. The choice of contours is not unique; choosing different contours for the same poles may result in different results, 
from which all the different solutions satisfy Maxwell equations at regular points, however only the right one satisfies all Maxwell equations at the singular point. Therefore, the verification is imperative.

From Eqs. (53) -(56), some identical differential properties between $\sin \left(k_{z m n} s\right) \Theta(s)$ and $Z_{\perp m n}(s)$, and between $\cos \left(k_{z m n} s\right) \Theta(s)$ and $Z_{\| m n}(s)$ can be found, so that

$$
\begin{aligned}
\frac{d}{d s} \psi_{\perp m n}(s)=k_{z m n} \psi_{z m n}(s), & (\mathrm{B} 1) \begin{array}{c}
i k_{z m n} E_{z m n}(\mathbf{r}), \text { Eq. (B2), and } \chi_{m n}=-i e \Pi_{z m n}^{-1} E_{z m n}^{*} \\
\text { have }
\end{array} \\
\nabla \cdot \mathbf{E} & =\sum_{m, n} \chi_{m n}\left[\nabla_{\perp} \cdot \mathbf{E}_{\perp m n}(\mathbf{r})\right] \psi_{\perp m n}(s)-\sum_{m, n} i \chi_{m n} E_{z m n}(\mathbf{r}) \frac{\partial}{\partial z} \psi_{z m n}(s) \\
& =\sum_{m, n} \chi_{m n}\left[i k_{z m n} E_{z m n}(\mathbf{r})\right] \psi_{\perp m n}(s)-\sum_{m, n} i \chi_{m n} E_{z m n}(\mathbf{r})\left[k_{z m n} \psi_{\perp m n}(s)-\delta(s)\right]=\delta(s) \sum_{m, n} i \chi_{m n} E_{z m n}(\mathbf{r}) \\
& =\frac{e}{\varepsilon} \delta(s) \sum_{m, n} \Pi_{z m n}^{-1} \varepsilon E_{z m n}^{*}\left(\mathbf{r}_{0}\right) E_{z m n}(\mathbf{r})=\frac{e}{\varepsilon} \delta\left(\mathbf{r}-\mathbf{r}_{0}\right) \delta(s),
\end{aligned}
$$

where identity Eq. (17) is employed in the last step.

Similarly, from Eqs. (59) and (60), using $\nabla_{\perp}$. $\mathbf{H}_{\perp m n}(\mathbf{r})=i k_{z m n} H_{z m n}(\mathbf{r}), \quad$ Eq. $\quad(\mathrm{B} 2), \quad$ and $\quad \chi_{m n}=$ $-i e \Pi_{z m n}^{-1} E_{z m n}^{*}\left(\mathbf{r}_{0}\right)$, we have

$$
\nabla \cdot \mathbf{H}=e \delta(s) \sum_{m, n} \Pi_{z m n}^{-1} E_{z m n}^{*}\left(\mathbf{r}_{0}\right) H_{z m n}(\mathbf{r})=0,
$$

where identity Eq. (18) is used.

So far, we have shown that the solution satisfies the two Maxwell divergence equations. One might be puzzled by the fact that $\mathbf{E}_{\perp}$ and $\mathbf{H}_{\perp}$, and $E_{z}$ and $H_{z}$ have the same mode expansion coefficients but lead to $\nabla \cdot \mathbf{E} \neq 0$ while $\nabla \cdot \mathbf{H}=0$. This is ascribed to the two identities given in Eqs. (17) and (18), which characterize the essential intrinsic difference between electric and magnetic fields.

The verification for curl equations is a little more complicated. Curl $\mathbf{H}$ can be written as $\nabla \times \mathbf{H}=(\nabla \times \mathbf{H})_{\perp}+$ $(\nabla \times \mathbf{H})_{z}$. With the help of Eqs. (2), (3), and (B1), we have

$$
\begin{aligned}
(\nabla \times \mathbf{H})_{\perp} & =\nabla_{\perp} \times \mathbf{H}_{z}+\nabla_{z} \times \mathbf{H}_{\perp} \\
& =\sum_{m, n} \chi_{m n} \omega_{m n} \varepsilon \mathbf{E}_{\perp m n}(\mathbf{r}) \psi_{z m n}(s) \\
& =\varepsilon \frac{\partial}{\partial t} \sum_{m, n} \chi_{m n} \mathbf{E}_{\perp m n}(\mathbf{r}) \psi_{\perp m n}(s)=\varepsilon \frac{\partial \mathbf{E}_{\perp}}{\partial t},
\end{aligned}
$$

where the mode phase velocity $v_{\mathrm{ph}}=\omega_{m n} / k_{z m n}$ is set to be equal to the particle velocity $v$.

Using Eq. (4), Eq. (B2), and identity Eq. (17), we have

$$
\begin{aligned}
(\nabla \times \mathbf{H})_{z}= & \nabla_{\perp} \times \mathbf{H}_{\perp}=\sum_{m, n} i \chi_{m n} \omega_{m n} \varepsilon \mathbf{E}_{z m n} \psi_{\perp m n}(s) \\
= & \varepsilon \frac{\partial}{\partial t} \sum_{m, n}-i \chi_{m n} \mathbf{E}_{z m n}(\mathbf{r}) \psi_{z m n}(s) \\
& +v \delta(s) \varepsilon \sum_{m, n} i \chi_{m n} \mathbf{E}_{z m n}(\mathbf{r}) \\
= & \varepsilon \frac{\partial \mathbf{E}_{z}}{\partial t}+\hat{\mathbf{z}} v e \delta\left(\mathbf{r}-\mathbf{r}_{0}\right) \delta(s)
\end{aligned}
$$

Inserting Eqs. (B5) and (B6) into $\nabla \times \mathbf{H}=(\nabla \times$ $\mathbf{H})_{\perp}+(\nabla \times \mathbf{H})_{z}$ yields $\nabla \times \mathbf{H}=\mathbf{J}+\varepsilon \partial \mathbf{E} / \partial t$.

The verification for $\nabla \times \mathbf{E}=-\mu \partial \mathbf{H} / \partial t$ is similar. The main difference is that identity Eq. (18) will be used this time. This is because $\nabla \cdot \mathbf{E}=\rho / \varepsilon$ is implicitly included in $\nabla \times \mathbf{H}=\mathbf{J}+\varepsilon \partial \mathbf{E} / \partial t$, while $\nabla \cdot \mathbf{H}=0$ is included in $\nabla \times \mathbf{E}=-\mu \partial \mathbf{H} / \partial t$.

In principle, satisfaction of Maxwell's equations for a solution is a sufficient condition for validity of the wave equations and the Poynting theorem $-\mathbf{J} \cdot \mathbf{E}=\nabla \cdot(\mathbf{E} \times$ $\mathbf{H})+\partial / \partial t[0.5(\varepsilon \mathbf{E} \cdot \mathbf{E}+\mu \mathbf{H} \cdot \mathbf{H})]$. Nevertheless, as an example, the verification for $E_{z}$-wave equation will be given below. From Eqs. (1) and (58), we have

$$
\begin{aligned}
\left(\nabla^{2}-\mu \varepsilon \frac{\partial^{2}}{\partial t^{2}}\right) E_{z}= & \left(v^{2} \mu \varepsilon-1\right) \sum_{m, n} i \chi_{m n} E_{z m n}(\mathbf{r}) \\
& \times\left(\frac{\partial^{2}}{\partial s^{2}}+k_{z m n}^{2}\right) \psi_{z m n}(s) .
\end{aligned}
$$

From Eq. (55), we have

$$
\left(\frac{d^{2}}{d s^{2}}+k_{z m n}^{2}\right) \psi_{z m n}(s)=\delta^{\prime}(s),
$$

where $\sin \left(k_{z m n} s\right) \delta(s)=0$ and $\cos \left(k_{z m n} s\right) \delta^{\prime}(s)=\delta^{\prime}(s)$ are employed [20].

Inserting Eq. (B8) into Eq. (B7), using Eq. (17) and (61), we arrive at the $E_{z}$-wave equation

$$
\begin{aligned}
\nabla^{2} E_{z}-\mu \varepsilon \frac{\partial^{2} E_{z}}{\partial t^{2}} & =\frac{e}{\varepsilon}\left(v^{2} \mu \varepsilon-1\right) \delta\left(\mathbf{r}-\mathbf{r}_{0}\right) \delta^{\prime}(s) \\
& =\frac{1}{\varepsilon} \frac{\partial \rho}{\partial z}+\mu \frac{\partial J_{z}}{\partial t} .
\end{aligned}
$$

From the above we see that the solution given in Eqs. (57)-(60) does satisfy all Maxwell equations, both at regular and singular points. In the proof, only the common eigenmode properties including two identities are employed, independently of the structure's transverse ge- 
ometry. This makes sense because the geometry of the DLW structure is already characterized by its 2D eigenfunctions.

\section{APPENDIX C: EIGENMODE AND SUPERPOSITION METHODS}

It is not easy to directly identify the difference between the solutions obtained from eigenmode and superposition methods in complicated calculations of wakefields, where both methods give series solutions made up of eigenfunctions. However, it might be helpful to know the difference; for example, the eigenfunctions from the two methods are not the same. In this appendix, the two methods are briefly reviewed and the difference is clearly shown through a simple mathematical example.

In the eigenmode method, eigenvalues, eigenfunctions, and orthogonality are the basic issues. For the DLW configuration, the transverse boundaries result in discrete eigenvalues, the eigenfunctions satisfy homogeneous 2D wave equations and they have continuous first-, secondorder derivatives except for on the boundaries. The orthogonality conditions are indispensable to get mode coefficients in solving wave equations.

In the superposition method used for the DLW accelerator structures $[3,4]$, a particular solution to an inhomogeneous wave equation in infinite space (infinite-space Green's function) is first obtained. Because the particular solution does not satisfy transverse boundary conditions, a general solution to the corresponding homogeneous equation is added to the particular solution so that the boundary conditions can be imposed, resulting in a set of coefficients for the general solution. This technique has a clear physical explanation: the total field is a linear superposition of the point-charge field and the induced field by boundaries. However, the solution obtained is not made up of traditional eigenfunctions because it explicitly contains a singularity at the transverse particle location [3,4], while in the eigenmode method the solution is made up of eigenfunctions that are analytical at any locations except on boundaries.

Let us take a simplified example to get a better understanding of the difference between the two methods. Suppose that there is a boundary-value problem, given by

$$
\begin{aligned}
d^{2} \phi / d x^{2}+a^{2} \phi & =-\delta(x), \\
\phi(-L) & =\phi(L)=0 \quad(a>0, L>0) .
\end{aligned}
$$

The solution from the superposition method is given by

$$
\phi(x)=\frac{1}{2 a}[\tan (a L) \cos (a x)+\sin (a x)]+\phi_{\text {part }}(x),
$$

where the first term comes from the general solution to $\phi^{\prime \prime}+a^{2} \phi=0$ and the second term is the particular solution given by $\phi_{\text {part }}(x)=-(1 / a) \sin (a x) \Theta(x)$.
The solution from the eigenmode method is given by

$\phi(x)=\sum_{m=0}^{+\infty} \frac{\cos \left(k_{m} x\right)}{L\left(k_{m}^{2}-a^{2}\right)}, \quad$ with $k_{m}=(2 m+1) \pi /(2 L)$.

We find that the solution Eq. (C2) has a singularity at $x=0$ where the first- and second-order derivatives do not exist in the sense of classic analysis theory, while the eigenfunction $\cos \left(k_{m} x\right)$ in the solution Eq. (C3) is analyti$\mathrm{cal}$, and of course, the singularity is hidden in the sum over infinite terms.

[1] T.-B. Zhang, T. C. Marshall, and J. L. Hirshfield, IEEE Trans. Plasma Sci. 26, 787 (1998).

[2] S. Y. Park and J. L. Hirshfield, Phys. Rev. E 62, 1266 (2000).

[3] K-Y. Ng, Phys. Rev. D 42, 1819 (1990).

[4] M. Rosing and W. Gai, Phys. Rev. D 42, 1829 (1990).

[5] S. Y. Park, C. Wang, and J. L. Hirshfield, in Advanced Accelerator Concepts: 10th Workshop, edited by Christopher E. Clayton and Patrick Muggli, AIP Conf. Proc. No. 647 (AIP, New York, 2002), pp. 527-541.

[6] T-B. Zhang, J. L. Hirshfield, T. C. Marshall, and B. Hafizi, Phys. Rev. E 56, 4647 (1997).

[7] A. Tremaine, J. Rosenzweig, and P. Schoessow, Phys. Rev. E 56, 7204 (1997).

[8] L. Xiao, W. Gai, and X. Sun, Phys. Rev. E 65, 016505 (2002).

[9] C. Wang, J. L. Hirshfield, J.-M. Fang, and T. C. Marshall, Phys. Rev. ST Accel. Beams 7, 051301 (2004).

[10] C. Wang, T.C. Marshall, V.P. Yakovlev, and J.L. Hirshfield, in Proceedings of the Particle Accelerator Conference, Knoxville, TN, 2005 (IEEE, Piscataway, NJ, 2005).

[11] W. Gai, P. Schoessow, B. Cole, R. Konecny, J. Norem, J. Rosenzweig, and J. Simpson, Phys. Rev. Lett. 61, 2756 (1988).

[12] B. M. Bolotovskii, Usp. Fiz. Nauk 75, 295 (1961) [Sov. Phys. Usp. 4, 781 (1962)].

[13] W. R. Smythe, Static and Dynamic Electricity (McGrawHill, NY, 1968), Chap. 13.

[14] R. E. Collin, Field Theory of Guided Waves (IEEE, NY, 1991), 2nd ed., Chap. 6.

[15] J. M. Dawson, Rev. Mod. Phys. 55, 403 (1983).

[16] LL G. Chambers, Br. J. Appl. Phys. 4, 39 (1953).

[17] C. Wang, V. P. Yakovlev, and J. L. Hirshfield, in Advanced Accelerator Concepts: Eleventh Workshop, edited by V. Yakimenko, AIP Conf. Proc. No. 737 (AIP, New York, 2004), pp. 871-879.

[18] For an external rf excitation, the field is expanded in terms of equifrequency eigenmodes and the flowing mode power is scaled to $\int\left(\mathbf{E}_{\perp m n} \times \mathbf{H}_{\perp m n}^{*}\right) \cdot \hat{\mathbf{z}} d S$, without the factor $\left(\nu^{2} \mu \varepsilon-1\right)$.

[19] A. V. Burov and A. V. Novokhatski, BINP Report No. 92$17,1992$.

[20] $\int_{-\infty}^{+\infty} f(s) \cos \left(k_{z m n} s\right) \delta^{\prime}(s) d s=\int_{-\infty}^{+\infty} f(s) \delta^{\prime}(s) d s=-f^{\prime}(0)$ leads to $\cos \left(k_{z m n} s\right) \delta^{\prime}(s)=\delta^{\prime}(s)$. 